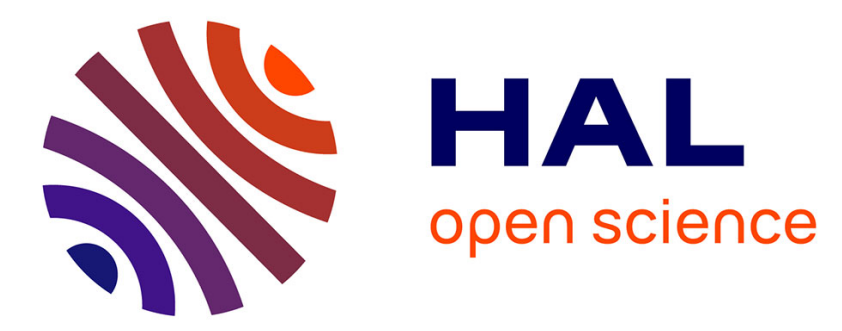

\title{
Size-velocity correlations in high order moment methods for polydisperse evaporating sprays: modeling and numerical issues
}

Aymeric Vié, Frédérique Laurent, Marc Massot

\section{To cite this version:}

Aymeric Vié, Frédérique Laurent, Marc Massot. Size-velocity correlations in high order moment methods for polydisperse evaporating sprays: modeling and numerical issues. Journal of Computational Physics, 2013, 237, pp.177-210. 10.1016/j.jcp.2012.11.043 . hal-00626869v3

HAL Id: hal-00626869

https://hal.science/hal-00626869v3

Submitted on 6 Aug 2012

HAL is a multi-disciplinary open access archive for the deposit and dissemination of scientific research documents, whether they are published or not. The documents may come from teaching and research institutions in France or abroad, or from public or private research centers.
L'archive ouverte pluridisciplinaire HAL, est destinée au dépôt et à la diffusion de documents scientifiques de niveau recherche, publiés ou non, émanant des établissements d'enseignement et de recherche français ou étrangers, des laboratoires publics ou privés. 


\title{
Size-velocity correlations in high order moment methods for polydisperse evaporating sprays: modeling and numerical issues
}

\author{
Aymeric Viéa $^{a, b}$, Frédérique Laurent ${ }^{\mathrm{a}, \mathrm{b}}$, Marc Massot ${ }^{\mathrm{a}, \mathrm{b}, \mathrm{c}}$ \\ ${ }^{a}$ CNRS, UPR 288, Laboratoire d'Energétique moléculaire et macroscopique, combustion, Grande Voie des Vignes, 92295 \\ Chatenay-Malabry, France \\ ${ }^{b}$ Ecole Centrale Paris, Grande Voie des Vignes, 92295 Chatenay-Malabry, France \\ ${ }^{c}$ Center for Turbulence Research, Stanford University, California 94305-4035, USA
}

\begin{abstract}
Kah et al. (2010) recently developed the Eulerian Multi-Size Moment model (EMSM) which tackles the modeling and numerical simulation of polydisperse multiphase flows. Using a high order moment method in a compact interval, they suggested to reconstruct the number density function (NDF) by Entropy Maximization, which leads to a unique and realizable NDF, potentially in several size intervals, thus leading to an hybrid method between Multifluid and high order. This reconstruction is used to simulate the evaporation process, by an evaluation of the flux of droplet disappearance at zero size, the fluxes of droplets between size intervals, and an accurate description of the size shift induced by evaporation (Massot et al. 2010). Although this method demonstrated its potential for evaporating polydisperse flows, two issues remain to be addressed. First, the EMSM only considers one velocity for all droplets, thus decoupling size from velocity, which is too restrictive for distributions with a large size spectrum. In most applications size-conditioned dynamics have to be accounted for. Second, the possibility to have separated dynamics for each size can lead to quasi-monodisperse distributions, which corresponds to a hard limiting case for the EM algorithm. So the behavior of the algorithm needs to be investigated, in order to reproduce the entire moment space with a reasonable accuracy. The aim of this paper is thus twofold. The EM and its related algorithm are enhanced by using a more accurate integration method in order to handle NDF close to the frontier of the moment space associated with an adaptive number of parameters to reconstruct the NDF accurately and efficiently, as well as tabulated initial guess to optimize the computational time. Then, a new model called CSVM (Coupled Size-Velocity Moments model) is introduced. Size-velocity correlations are addressed either in the evaporation and drag processes, or in the convective transport. To reach this goal, a velocity reconstruction for each size is suggested, using only one additional moment per dimension, and which can be directly applied to several size intervals. Thus, this method is a direct generalization of EMSM. To handle the convective transport, a flux splitting scheme is proposed, based on the underlying kinetic description of the disperse phase. Comparing to existing approaches, a main novelty of the CSVM is that our kinetic approach ensures built-in realizability conditions, no additional corrections of the moments being needed at each time step. The full strategy is first evaluated in 0D and 1D cases, which either demonstrates the ability to reproduce both evaporation, drag force and convection with size-velocity correlations, or the possible extension to several size intervals. Finally, the method is applied on 2D cases with only one section, showing the ability of the CSVM and its related algorithms to capture the main physics of polydisperse evaporating sprays with a minimal number of moments.
\end{abstract}

Key words: Polydisperse sprays, high order moment method, Entropy Maximization, flux splitting, size-velocity correlations

Email addresses: aymeric.vie@ecp.fr (Aymeric Vié), frederique.laurent@ecp.fr (Frédérique Laurent), marc.massot@ecp.fr (Marc Massot) 


\section{Introduction}

Multiphase flows occur in several industrial applications, such as internal combustion engines $([1,2]$ and references therein), gas turbines [3] or rocket boosters [4]. Those applications are linked by the existence of a disperse liquid phase, composed of droplets of various sizes. Simulating this disperse phase accurately becomes crucial, as it highly influences the global behavior of the full device (consumption, overall power, pollutant emissions, etc...).

The description of a disperse phase may rely on a population balance equation (PBE) on the number density function (NDF), namely the Williams-Boltzmann equation in the context of sprays $[5,6]$. The PBE describes the time evolution of the NDF in the real space (position) and in the phase space determined by the chosen internal coordinates for the description of the NDF (size, velocity, temperature...). Simulating the PBE can be achieved by using the Lagrangian direct simulation Monte-Carlo method (DSMC [7]), which solves Lagrangian equations for a large sample of particles, in order to reach converged statistics. This approach is considered as the most accurate, but is very expensive, especially for unsteady flows, and is not well adapted for high performance parallel computing due to load balancing as well as for coupling to the gas phase due to interpolations between gas and disperse phases [8]. Its use for industrial applications stays unreachable, even with the great increase in computational resources. Eulerian methods can overcome this problem by not directly solving the PBE on the NDF, but its moments, which are integrated quantities over phase space. In the idealized case of monodisperse distributions, i.e. when all droplets have the same size, the NDF can be easily reconstructed because only two moments are necessary. But in the case of polydisperse distributions, an infinite number of moments is needed to fully determine the NDF, which is called the Hausdorff moment problem [9] if a compact support is considered. With fewer moments, the NDF is not uniquely determine (the finite Hausdorff moment problem), and additional constraints are needed to obtain a one-to-one relationship between the moments and the NDF.

To account for size polydispersion, three ways can be envisaged:

1. A size phase space discretization: the size space can be discretized into intervals called "sections" [10], which leads to a Finite Volume formulation called Multifluid approach in the context of sprays [11],

2. A quadrature-based moment method : a limited set of moments is used to build a quadrature approximation of the NDF $[12,13]$,

3. A moment method with continuous reconstruction: a limited set of size moments is used to reconstruct the NDF continuously $[14,15,16,17,18,19]$.

Discretizing the size phase space is the simplest way to capture the size polydispersion. A first order Multifluid approach, which uses one moment per section is easy to implement and accurate [20]. But in order to limit diffusion in size space, a high number of sections is needed, thus increasing the computational cost [21]. Using two moments per section, second order Multifluid approaches can reduced the number of sections needed to reach a high accuracy $[22,21,4]$, but one may want to further minimize the number of sections. Adopting a moment point of view leads to the transport of only one "fluid", but with several moments. This can be done using QMOM (quadrature method of moment) [12] or DQMOM (direct quadrature method of moment) [13]. These approaches are linked by the reconstruction of the NDF : a sum of Dirac $\delta$-functions evolving jointly. The difference is that QMOM solves equations on the moment set, whereas DQMOM directly solves the equation on weights and abscissas of the Dirac $\delta$-functions. Those two methods have shown their potential for coalescence or breakage, but encounter difficulties for evaporating spray, especially with continuous distributions, due to the disappearance flux of droplets at zero size [23]. Actually, the lower order representation of the QMOM or the DQMOM is not able to reproduce this continuous flux, the disappearance of droplet being possible only when a Dirac $\delta$-function reaches the zero abscissa, and leading to a singular flux.

To solve this intrinsic problem of the representation of the NDF, Kah et al. [2, 15] have suggested a new strategy, called Eulerian Multi-Size Moment model (EMSM), taking advantage of a continuous description of the NDF to determine the flux at zero size, and the evaluation of the shift in size induced by evaporation by a combined flux/quadrature approach. The reconstruction of the NDF is done by Entropy Maximization (EM) [24]. This is a convex optimization problem with constraints, which admits a unique solution, as long 
as the moment vector stays in the interior of the moment space [25]. This NDF is used to compute the flux at zero size, and the evaporation is evaluated by means of the zero flux and a quadrature approximation. The potential of EMSM is demonstrated in [26], where it is shown that the computational time for a equivalent accuracy is clearly better than the Multifluid method. Furthermore, the EMSM can be coupled to a discretization of the size space, leading to a hybrid method between Multifluid and high order moment approaches $[15,2]$.

The evolution in size phase space solved, an important issue to be tackled is the evolution in physical space. In fact this issue is twofold: we need to define a closure in velocity phase space, which will depend on the number of velocity moments we want and which relates to the expected dynamics, and then we need to build the numerical scheme used to transport the set of moments in physical space. In the current study we will focus on flow regimes for which no droplet trajectory crossings occur, which, following [27] in the case of turbulent flows, corresponds to Stokes number in the range [0,1]. For high Stokes number, statistical [28], deterministic $[29,30]$ or hybrid $[29,31]$ methods can be used, but are out the scope of the present work. Concerning the definition of the numerical scheme, in general a first order Finite Volume scheme is able to transport a set of moments vector, preserving the moment space. But for complex problems, high order methods are needed. As shown in [32], classical high order finite volume numerical schemes cannot always keep the moments in the interior of the moment space. To overcome this difficulty, a kinetic scheme adapted to a high number of moments is proposed in [26], which uses a linear reconstruction on the canonical moments, and intrinsically preserves the realizability of the moment set.

The EMSM associated with a stable and realizable kinetic scheme for the physical transport is a good candidate for the simulation of complex flows and has already been implemented in an industrial code (IFPC3D [33, 34]). But two important issues still remain unsolved. Firstly, the EMSM uses the same velocity for all droplets, independently of their size. This is quite a strong assumption when drag force is accounted for, as it may generate size-conditionned differential dynamics. The effect of such an assumption was illustrated in a test case proposed in [35] which consists in the injection of droplets into a gaseous crossflow at constant and uniform velocity, with a constant size distribution for which Stokes numbers based on the convective time of the gas phase range in $[0.083,0.744]$. The flow field was solved using the AVBP code [36], using two formalisms: a two-fluid approach, which is equivalent to the EMSM in the case of non-evaporating flows, and a Multifluid approach with 10 sections. Results are shown in Fig. 1 . When only one velocity is considered for the whole distribution, only one trajectory can be reproduced. This effect is partially accounted for, using a Multifluid approach, because each section has its proper velocity. But for a coarse size discretization, such as the ten section example given in Fig. 1, this leads to distinct trajectories separated by vacuum regions. Secondly, the ability to capture separated dynamics depending on the droplet size would generate quasimonodisperse distributions. Unfortunately, the EMSM and its related EM algorithm encounter difficulties in reproducing this kind of NDF. It limits the applicability of the EM or at least introduces an error for this type of NDF [15]. This type of highly segregative flows is typical of swirling injection [14] in aeronautical configurations, even if the turbulent mixing tends to generate continuous and regular NDFs.

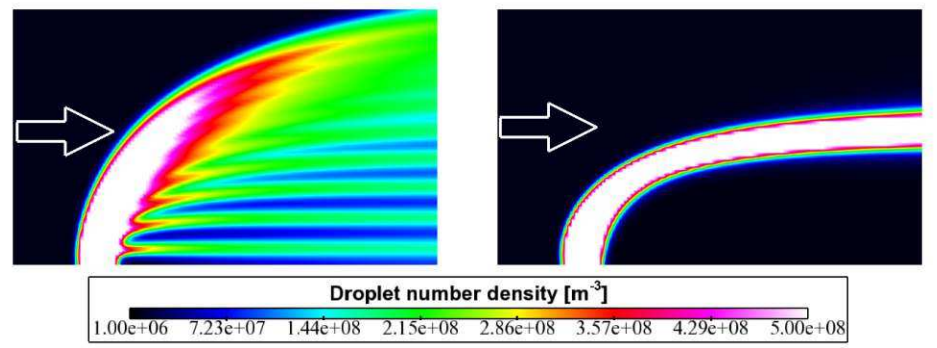

Figure 1: 2D Crossflow: Multifluid method with 10 sections (left) and to a monodisperse-monokinetic approach equivalent to the EMSM in this non-evaporating case (right). Stokes numbers are in the range $[0.083,0.744]$ for the Multifluid method and 0.332 for the monokinetic approach [35]. 
The developments of the present work address these issues, in terms of modeling and numerics:

- Compared to existing reconstructions $[16,14,19,37]$, the EM is for now the only reconstruction strategy that enables to cover the whole moment space (ie. all possible combinations of moments that corresponds to a positive NDF), excepting its frontier which can be reached as close as necessary. Considering the propensity of size-velocity correlations to generate moment sets at the frontier of the moment space, we enhance the EM numerics using adaptive procedures, in terms of integration methods and controlled moments, and a tabulated initial guess ${ }^{1}$,

- A new method is developed, called Coupled Size-Velocity Moments model (CSVM), that takes into account size-velocity correlations by the transport of an additional size-velocity moment for each space dimension, which allows to reconstruct the velocity against size. Comparing to the velocity reconstruction of Dems et al. [16] based on the work of Ferry and Balachandar [38, 39], which assumes a linear expansion of the disperse phase velocity around the gas phase velocity and uses only one size-velocity moment, our reconstruction uses at least two size-velocity moments, to be able to capture inversion of the relative velocity with respect to size which may occur in turbulent vortical flows. Moreover, in the spirit of the EMSM, the CSVM is adapted to a discretization in size phase space into size intervals called sections (such as in the Multifluid point of view), making possible to increase the accuracy either by increasing the number of moments per section or by increasing the number of sections. The hybrid character of the method is a major advantage and leads to a very flexible method.

- The evolution in size space for the size moments is done by the determination of the disappearance flux of droplets coupled to a size quadrature approximation. But, contrary to the initial EMSM where the evolution of velocity moments is straightforward due to the fact that all droplet size have the same velocity, the newly introduced size-velocity moments also evolve in size space using both size and velocity reconstructions. The evolution in velocity space due to drag force is done using a CQMOM quadrature [40] coupled to EMSM, which permits to account for the coupled size and velocity evolution, by integrating ordinary differential equations. Using a quadrature approach for the evolution in phase space is important, because complex laws for drag force, evaporation and heating can be easily accounted for, even if we consider simple laws in this work [15].

- A new numerical kinetic scheme is introduced for the convection in physical space, which is based on the flux splitting technique, and preserves the moment space. Compared to the transport scheme in Beck et al. [18] and Dems et al. [16], for which each moment is transported using its proper momentaverage velocity, our scheme directly relies on the kinetic representation of the NDF, which allows to keep a accurate description of the fluxes as well as the realizability of the moments.

This new moment method is then assessed in 0D for both evaporating and non-evaporating cases and is shown to capture size-conditioned dynamics even in the configuration of oscillating gaseous flow field and in the presence of a large spectrum of sizes. This transport scheme is evaluated in $1 \mathrm{D}$ cases, its order studied and its robustness demonstrated. We then switch to two extreme non-evaporating cases in order to isolate the size-velocity correlations due to drag force and convection, the evaporation part having already been characterized in the framework of EMSM studies $[15,26]$. We study a crossflow case and a Taylor-Green case, with steady gaseous velocity fields, where a strong size-conditioned dynamics leads to moment vectors very close to the frontier of the moment space. Comparisons with either analytical solutions or other solvers such as Lagrangian, Multi-fluid or EMSM show the potential and the accuracy of the method. Eventually, in order to provide a test case closer to more realistic unsteady configurations, we investigate a weakly turbulent free jet with a very large size spectrum in a non-evaporating configuration. An excellent agreement is obtained compared to Lagrangian and Multifluid with 10 sections, thus completing the validation of the method. We finally provide comments on the memory storage and computational cost of the method as well as on its application to industrial configurations and potential extensions.

\footnotetext{
${ }^{1}$ This study is mandatory for size-velocity correlations, but can also be seen as an autonomous topic, as it can be useful for other applications that uses EM.
} 
The paper is organized as follows. First, the EMSM is described, with its advantages and drawbacks in Section 2. Then improvements of the EM are presented in Section 3, in order to be able to reproduce the whole moment space with a controlled error. The CSVM which takes into account size-velocity correlations is introduced in Section 4. Concerning the evolution in phase space and the transport in physical space, the drag/evaporation strategy and the flux splitting kinetic scheme are detailed in section 5 and evaluated in 0D/1D cases in section 6 . Finally, an evaluation in 2D extreme cases of crossflow and Taylor-Green vortices is investigated in section 7 as well as the final free jet configuration.

\section{The Eulerian Multi-Size Moment model}

\subsection{The moment problem}

We consider dilute sprays at high Knudsen numbers, so that there is no effect of the disperse phase on the gas phase and collisions can be considered as negligible. The phase space of the NDF is limited to droplet surface $S$ and velocity $v$ in one dimension, leading to the following PBE:

$$
\frac{\partial f}{\partial t}+v \frac{\partial f}{\partial x}+\frac{\partial}{\partial v}\left(\frac{v-u_{g}}{\tau_{p}} f\right)+\frac{\partial R_{S} f}{\partial S}=0,
$$

where $R_{S}=\mathrm{d} S / \mathrm{d} t$ is the evaporation rate which is constant in the case of the $d^{2}$ evaporation law and $\tau_{p}$ is the relaxation time of the droplets for Stokes' drag. Using non-dimensional variable $S^{*}=S / S_{\max }, t^{*}=t / \tau_{g}$, $v^{*}=v / v_{\text {ref }}$ where $S_{\max }$ is the maximum droplet surface, $\tau_{g}$ and $v_{\text {ref }}$ a reference time scale and velocity of the gaseous flow, we get the non-dimensional PBE:

$$
\frac{\partial f}{\partial t^{*}}+v^{*} \frac{\partial f}{\partial x^{*}}+\frac{\partial}{\partial v^{*}}\left(\frac{v^{*}-u_{g}^{*}}{\mathrm{St}} f\right)+\frac{\partial R_{S}^{*} f}{\partial S^{*}}=0
$$

where St $=\tau_{p} / \tau_{g}$ is the Stokes number. For sake of clarity, star exponent will be dropped for non-dimensional variables. To solve Eq. 2, we look at the moments of the NDF. In one dimension, and considering the compact support $[0,1]$ for the size space (which corresponds to $\left[0, S_{\max }\right]$ in dimensional quantities), these are defined by:

$$
M_{i}^{l}(t, x)=\int_{-\infty}^{\infty} \int_{0}^{1} S^{l} v^{i} f(t, x, v, S) \mathrm{d} v \mathrm{~d} S .
$$

The equation for $M_{i}^{l}(t, x)^{2}$ is then:

$$
\begin{aligned}
\frac{\partial M_{i}^{l}}{\partial t}+\frac{\partial M_{i+1}^{l}}{\partial x}= & -\int_{0}^{1} i \frac{S^{l}}{\mathrm{St}}\left(v^{i}-u_{g} v^{i-1}\right) f \mathrm{~d} S \mathrm{~d} v \\
& +R_{S} M_{i}^{l-1}+R_{S}\left[\int_{-\infty}^{\infty} v^{i} S^{l} f(t, x, v, S) \mathrm{d} v\right]_{S=0}^{S=1}
\end{aligned}
$$

In Eq. 4, the main issues are the modeling of the second left hand side term (convection) and the third right hand side term (evaporation). The former is due to the fact that with a finite set of moments, the flux for the highest order moment equation is unclosed. The latter requires the knowledge of point-wise values of the NDF at the edge of the size phase space. In the following, one section is considered, but the whole method can be easily adapted to a Multifluid approach by means of a change of variables [15].

\footnotetext{
${ }^{2}$ Exponent $l$ stands for the order in size of the moment, and not for a power.
} 


\subsection{The moment space}

Even if the moment space where lives the size moment vector $\mathcal{M}_{0}=\left(M_{0}^{0}, M_{0}^{1}, \ldots, M_{0}^{N}\right)$ is convex, it has a complex geometry in the semi-open space $\mathbb{R}_{+}^{N+1}[15]$. The normalized moment vector $\left(M_{0}^{1} / M_{0}^{0}, \ldots, M_{0}^{N} / M_{0}^{0}\right)$ lives in a closed convex space of $[0,1]^{N}$, but still has a complex geometry. A simpler space can be determined by using the canonical moments [25]. The first four canonical moments are:

$$
\begin{aligned}
p_{0} & =1 \\
p_{1} & =\frac{M_{0}^{1}}{M_{0}^{0}}, \\
p_{2} & =\frac{M_{0}^{0} M_{0}^{2}-\left(M_{0}^{1}\right)^{2}}{M_{0}^{1}\left(M_{0}^{0}-M_{0}^{1}\right)}, \\
p_{3} & =\frac{\left(M_{0}^{0}-M_{0}^{1}\right)\left(M_{0}^{1} M_{0}^{3}-\left(M_{0}^{2}\right)^{2}\right)}{\left(M_{0}^{0} M_{0}^{2}-\left(M_{0}^{1}\right)^{2}\right)\left(M_{0}^{1}-M_{0}^{2}\right)} .
\end{aligned}
$$

So the actual moments reads:

$$
\begin{aligned}
& M_{0}^{1}=M_{0}^{0} p_{1} \\
& M_{0}^{2}=M_{0}^{0} p_{1}\left[\left(1-p_{1}\right) p_{2}+p_{1}\right] \\
& M_{0}^{3}=M_{0}^{0} p_{1}\left[\left(1-p_{1}\right)\left(1-p_{2}\right) p_{2} p_{3}+\left[\left(1-p_{1}\right) p_{2}+p_{1}\right]^{2}\right] .
\end{aligned}
$$

The canonical moments live in the full cube $[0,1]^{N}$, leading to simpler analysis, especially in terms of realizability of the moment vector. The frontier of the moment space is defined by the values 0 or 1 for one of the canonical moment $p_{k}$. This frontier is characterized by the existence of a unique solution of the Hausdorff moment probem: a NDF which is a sum of weighted Dirac $\delta$-functions $f(S)=\sum_{k=1}^{2} w_{k} \delta\left(S-S_{k}\right)$.

In the interior of the moment space where canonical moments are neither equal to 0 nor 1 , there is an infinity of NDF, the moments of which are a finite moment vector.

The EMSM tackles the problem of evaporation terms by a reconstruction of the NDF. In this work we will considered 4 moments in size and one size interval, but the method can be extended to more moments and more size intervals. Considering that the velocity does not depend on the size, the NDF can be decomposed in the following way:

$$
f(t, x, v, S)=n(t, x, S) \delta(v-U(t, x)),
$$

where $U=M_{1}^{0} / M_{0}^{0}$. The EMSM reconstructs $n(t, x, S)$ using EM [24]. The Shannon Entropy is defined by:

$$
\mathbf{H}(f)=-\int_{0}^{1} n(t, x, S) \ln n(t, x, S) \mathrm{d} S .
$$

Associated with $N$ moments constraints, the maximization of $\mathbf{H}(f)$ imposes the unique following reconstruction (for convenience $x$ and $t$ dependences are dropped, as they do not influence the reconstruction):

$$
n(S)=\exp \left(-\sum_{j=0}^{N} \zeta_{j} S^{j}\right),
$$

where $\zeta_{j}$ are Lagrange multipliers. The interested reader will refer to [24] and [9] and references therein for more information. The following convex potential is then minimized:

$$
\Delta=\int_{0}^{1}\left[\exp \left(-\sum_{j=0}^{N} \zeta_{j} S^{j}\right)-1\right] \mathrm{d} S+\sum_{j=0}^{N} \zeta_{j} M_{0}^{j} .
$$

Indeed, its stationary points are given by:

$$
\frac{\partial \Delta}{\partial \zeta_{i}}=0 \Rightarrow \int_{S_{\min }}^{S_{\max }} S^{i} \exp \left(-\sum_{j=0}^{N} \zeta_{j} S^{j}\right) \mathrm{d} S=M_{0}^{i} .
$$


Numerically, the non-linear system of Eq. 16 is solved using a Newton method. Starting form initial choices $\zeta=\left(\zeta_{0}, \ldots, \zeta_{N}\right)^{T}$, updated $\zeta^{T}$ is defined from:

$$
\zeta^{+}=\zeta-\frac{\mathcal{M}_{0}-\langle\mathcal{M}\rangle_{\zeta}}{H}
$$

where $\langle\mathcal{M}\rangle_{\zeta}=\left(\left\langle m^{0}\right\rangle_{\zeta}, \ldots,\left\langle m^{N}\right\rangle_{\zeta}\right)^{T}$ is the vector of approximated moments:

$$
\left\langle m^{i}\right\rangle=\int_{0}^{1} S^{i} \exp \left(-\sum_{j=0}^{N} \zeta_{j} S^{j}\right) d S
$$

and $H$ is the Hessian matrix defined by $H_{i, j}=\frac{\partial \Delta}{\partial \zeta_{i} \partial \zeta_{j}}=\left\langle m^{i+j}\right\rangle_{\zeta}$ for $i, j=0, . ., N$. Moments $\left\langle m^{i}\right\rangle$ for $i=0, . ., N$ are evaluated by a Gauss-Legendre quadrature method evaluates the integrals. In [15], it has been proven that this integration method with 24 quadrature points is sufficient to reach the subset $[0.01,0.99]^{3}$ of the canonical moment space and that it is sufficient to evaluate the disappearance flux of droplet at zero size with an error less than 1\%. Higher order moments that are needed for the Hessian matrix are computed using an integration by parts [41]:

$$
\left\langle m^{N+k}\right\rangle=\frac{1}{N \zeta_{N}}\left((k+1)\left\langle m^{k}\right\rangle-\sum_{l=1}^{N-1} l \zeta_{l}\left\langle m^{l+k}\right\rangle+W_{k}(1, \zeta)-W_{k}(0, \zeta)\right),
$$

where $W_{k}(a, \zeta)=a^{k+1} \exp \left(-\sum_{j=0}^{N} \zeta_{j} a^{j}\right)$

Starting from the reconstructed NDF, the evaluation of the evaporation process is made in two steps. First, the disappearance flux at zero size is evaluated, and corresponds to the part of the moment that will disappear during a time step $\Delta t$ :

$$
F_{i}^{l}=\int_{0}^{R_{S} \Delta t} U^{i} S^{l} n_{l}(S) \mathrm{d} S
$$

The moments are then corrected:

$$
\widetilde{M}_{i}^{l}=M_{i}^{l}-F_{i}^{l}
$$

The evolution in phase space is determined by a quadrature approach: given the quadrature on the corrected moments,

$$
\widetilde{M}_{i}^{l}(t)=U^{i} \sum_{k=1}^{2} w_{k} S_{k}(t)^{l}
$$

the evolution of abscissas is computed thanks to the evaporation law:

$$
\frac{d S_{k}}{d t}=R_{S} \Rightarrow S_{k}(t+\Delta t)=S_{k}(t)+R_{S} \Delta t
$$

so that:

$$
\widetilde{M}_{i}^{l}(t+\Delta t)=U^{i} \sum_{k=1}^{2} w_{k}\left(S_{k}(t+\Delta t)\right)^{l},
$$

As stated in [15], this method can account for the $d^{2}$-law as well as any other evaporation law (if $R_{S}$ is evaluated at $S_{k}$ ) with a good accuracy and a rigorous preservation of the moment space.

For the transport in physical space, Kah et al. [26] developed a second order in time and space Finite Volume scheme based on a spatial reconstruction of the canonical moments, which preserves the moment space. The authors evaluated the full strategy on complex $2 \mathrm{D}$ configurations and have proven its ability to predict such evaporating polydisperse flows, as well as its efficiency compared to the Multifluid approach. 


\section{Numerical issues with Entropy Maximization}

In [15], the EM is used to quantify the disappearance flux of droplets. Such an algorithm was designed in order to mainly treat smooth distributions where the EM reconstruction associated with a Gauss-Legendre quadrature is able to reach a controlled precision of typically $10^{-6}$ on the evaporative flux, with the limit of a reasonable number of Newton steps. Even if some treatment was proposed in [15] at the frontier of the moment space, the method could only achieve a precision of $10^{-2}$ on the moments in the favorable context of size-velocity decoupling which was coherent with the expected level of modeling of that paper. Unfortunately, in the present context, where we reach a much higher accuracy in terms of size-velocity coupling and treatment of polydispersion, the frontier of the moment space will potentially have a much more important role in some zones of the flow and we need to upgrade the EM procedure in both precision and algorithmic efficiency in order to cope with the increase of modeling level.

Three new ingredients are introduced in the present section:

- In order to drastically increase the computational efficiency of the EM subroutine, a tabulated initial guess for the Newton solver has been implemented and we provide the key features of such an approach which is used in most of the interior of the moment space, that is in the cube $[0.1,0.9]^{3}$ in terms of the canonical moments (Sec. 3.1),

- An adaptive support for the evaluation of the integrals in the EM subroutine has been implemented, thus leading to an important gain in accuracy (Sec. 3.2.1),

- An adaptive reduction of the number of parameters required for the description of the moments vectors very close to the frontier of the moment space is implemented. It allows to reduce the computational time and to increase the precision compared to the initial algorithm in [15] (Sec. 3.2.2).

\subsection{Tabulation}

To reduce the number of iterations needed for the Newton solver to converge, the tabulation of the parameters $\left(\zeta_{k}\right)$ is considered. The evolution of the parameters against the canonical moments is first investigated. In Fig. $2, \zeta_{1}$ is plotted against $p_{2}$ and $p_{3}$ for $p_{1}=0.1,0.5,0.9$. Notice that $p_{2}$ and $p_{3}$ vary between 0.1 and 0.9 . In fact, the parameters evolve smoothly in the interior of the moment space. The tabulation will then give accurate results. The question is now to evaluate the error for a direct tabulation of the parameters.

Two interpolation methods are compared for the tabulation: a linear reconstruction and a third order polynomial reconstruction. The tabulation step is $\Delta p=0.01$ for each canonical moment, and the tabulation is done in the cube $[0.1,0.9]^{3}$.

On Tab. 1, the error made on the moments with the two methods are compared for different sets of canonical moments in the interior of the moment space. Each set is chosen to be as far as possible from tabulated values, to exhibit the maximal error. With a third order polynomial the error can be close to $10^{-4}$.

\begin{tabular}{|c|c|c|c|c|}
\hline$p_{1}$ & $p_{2}$ & $p_{3}$ & Linear & 3rd order \\
\hline 0.105 & 0.105 & 0.105 & $3.8 \mathrm{e}-3$ & $7.0 \mathrm{e}-5$ \\
0.505 & 0.505 & 0.505 & $3.3 \mathrm{e}-4$ & $2.9 \mathrm{e}-7$ \\
0.895 & 0.895 & 0.895 & $3.7 \mathrm{e}-3$ & $1.0 \mathrm{e}-4$ \\
0.505 & 0.105 & 0.105 & $4.3 \mathrm{e}-3$ & $4.8 \mathrm{e}-5$ \\
0.505 & 0.895 & 0.895 & $2.6 \mathrm{e}-3$ & $7.3 \mathrm{e}-5$ \\
\hline
\end{tabular}

Table 1: Error on moments using a linear or a third order interpolation method for five sets of canonical moments.

If a higher precision is required, the tabulation is used to define an initial guess for the Newton iterative solver, which is supposed to reduce the computational time needed to reach a given accuracy of $10^{-6}$. 

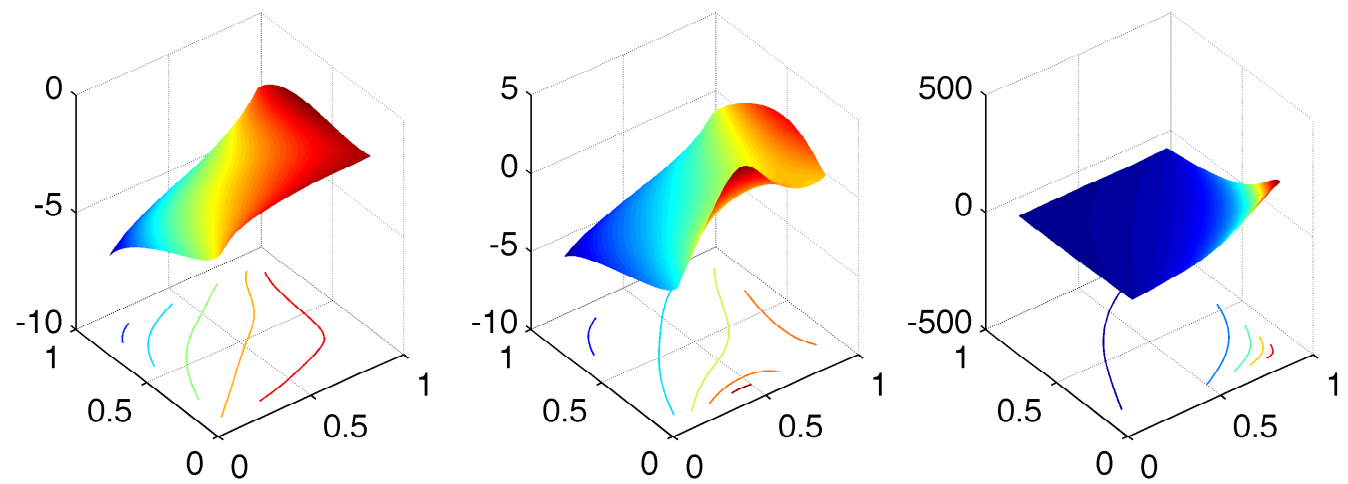

Figure 2: Entropy maximization parameters: $\zeta_{1}$ against canonical moments $p_{2}$ and $p_{3}$, with $p_{1}=0.1$ (left), $p_{1}=0.5$ (center), $p_{1}=0.9$ (right).

Table 2 shows the numbers of iterations needed by the Newton solver for five moments sets depending on the initial guess: a constant number density distribution, the nearest point in the tabulation, a linear or a $3^{r d}$ order interpolation. It can be seen that the tabulation of the initial guess lowers significantly the number of Newton iterations needed, the fastest method being the more accurate third order reconstruction for the initial guess ${ }^{3}$.

\begin{tabular}{|c|c|c|c|c|c|c|}
\hline$p_{1}$ & $p_{2}$ & $p_{3}$ & Constant & Nearest & Linear & 3rd order \\
\hline 0.105 & 0.105 & 0.105 & 12 & 4 & 2 & 1 \\
0.505 & 0.505 & 0.505 & 4 & 2 & 1 & 0 \\
0.895 & 0.895 & 0.895 & 17 & 3 & 2 & 1 \\
0.505 & 0.105 & 0.105 & 8 & 3 & 2 & 1 \\
0.505 & 0.895 & 0.895 & 10 & 3 & 2 & 1 \\
\hline
\end{tabular}

Table 2: Number of iterations of the Newton solver using a constant initial guess, or a tabulated initial guess with nearest point, with linear or third order interpolation method for five set of canonical moments.

\subsection{Dealing with the frontier of the moment space}

As stated in the previous section, the parameters $\zeta$ evolve slowly with the canonical moments in the subspace $[0.1,0.9]^{3}$. But close to the frontier, where at least one canonical moment is in $[0.0,0.1] \cup[0.9,1.0]$, the variation of $\zeta$ for a small variation of the canonical moments is very important. The tabulation technique cannot be used in this part of the moment space, where the convex minimization problem becomes illconditioned. However the initial guess for the Newton solver is chosen to be the closest point in $[0.1,0.9]^{3}$.

At the frontier of the moment space, the distribution is a sum of Dirac $\delta$-functions. For a four-moments reconstruction the frontier is defined as follow (see $[25,15]$ for more details):

- if $p_{1}=0$ or $p_{1}=1$ : the frontier corresponds to two points in the normalized moment space. The NDF is a $\delta$-function at $S=0$ (if $p_{1}=0$ ) or $S=1$ (if $p_{1}=1$ ).

\footnotetext{
${ }^{3}$ The interpolation step is negligible in terms of computational time, comparing to one Newton step.
} 
- if $p_{2}=0$ or $p_{2}=1$ : the frontier corresponds to two submanifolds of dimension one in the normalized moment space parametrized by $p_{1}$ for example and connecting the previous two points. The NDF is a $\delta$-function at $S=p_{1}$ (if $p_{2}=0$ ) or two $\delta$-functions with different weights at $S=0$ and $S=1$ (if $\left.p_{2}=1\right)$.

- if $p_{3}=0$ or $p_{3}=1$ : the frontier corresponds to two submanifolds of dimension two in the normalized moment space parametrized by $\left(p_{1}, p_{2}\right)$ for example and connecting the previous two curves. The $\mathrm{NDF}$ is a sum of two $\delta$-functions with different weights and surfaces, one of them being located at the boundary of the $[0,1]$ interval.

It is clear that the process is recursive and can be extended to higher order moments. In the present contribution we will work in the interior of the moment set, that is, we will never reach the frontier of the moment space with its various levels of degeneracy. However, even if we are working on the open moment space, the EM should possibly used as close as we want from the frontier of the moment space. Now being close to this frontier imposes two constraints on the Newton solver used for EM: the NDF approached a singular function constituted of a sum of Dirac $\delta$-functions, and the Hessian matrix becomes ill-conditioned. We thus introduce two strategies to handle the neighborhood of the frontier of the moment space: an adaptive support for the integrals and a way to choose the optimal number of EM parameters.

\subsubsection{Adaptive support for the integrals}

The reconstruction can exhibit very high variations for limiting cases near the frontier of the moment space. In such cases, the classical Gauss-Legendre quadrature may use abscissas where the function is close to zero, limiting the precision of the method. To overcome this problem, an adaptive support for the gaussian quadrature is used. This support is set for a given threshold $\epsilon_{0}$ in order to locate zones with negligible number density, by an analysis of the reconstructed $\operatorname{NDF} n(S)^{4}$ :

$$
n(S)=\exp \left(\sum_{j=0}^{N} \zeta_{j} S^{j}\right)=\epsilon_{0} \Longleftrightarrow \sum_{j=0}^{N} \zeta_{j} S^{j}-\ln \epsilon_{0}=0 .
$$

Finding the roots of the polynomial in Eq. (25) allows to define the integration intervals. As we considered four size moments in this work, the integration support will be made of one or two intervals (as the polynomial will have 3 real roots at most). In practice $\epsilon_{0}$ is set to the expected tolerance on the integrals evaluation (to reach an accuracy of $\epsilon$ on the integrals, $\epsilon_{0}$ is set to $\epsilon$ ).

The ability of the adaptive support to compute the integral of singular functions is assessed on the computation of the integral of $\exp (-K S)$ for which the analytical solution is easily found. In Fig. 3, the relative error made by both fixed and adaptive support Gauss-Legendre integration methods are shown. For $n(S)=\exp (-10 S)$, the two methods exhibit the same results, as the function extrema are larger than the threshold $\epsilon_{0}=5 \cdot 10^{-6}\left(n(S=0)=1\right.$ and $\left.n(S=1)=\exp (-10)=4.5 \cdot 10^{-5}\right)$, and the support stays [0,1] for the adaptive method. For $n(S)=\exp \left(-10^{2} S\right)$, the adaptive support is activated, as the extremum values are 1 at $S=0$ and $n(S=1)=\exp \left(-10^{2}\right)=3.7 .10^{-44}$. With $\epsilon_{0}=5.10^{-6}$, the adapted support is now $[0,0.398]$ and the accuracy of the adaptive method is considerably higher, even if the fixed support method is able to compute this integrals with high but reasonable number of nodes. For $n(S)=\exp \left(-10^{4} S\right)$, the fixed support method is not able to compute this integral, even with 40 nodes, where the adaptive method uses the support $\left[0,4.44 .10^{-3}\right]$ and computes the integral with an error less than $10^{-6}$ with only 15 nodes, and confirms the importance and the accuracy of the integration method.

\subsubsection{Adaptive reconstruction at the frontier of the moment space}

In the interior of the moment space, in a small neighborhood of the frontier, the Newton solver experiences a very slow convergence due to an ill-conditioned Hessian matrix. This difficulty is mainly encountered when

\footnotetext{
${ }^{4}$ This NDF can be the final reconstruction or an intermediate one in the Newton iterations, as the Hessian matrix needs to be reevaluated at each iteration with the new parameters.
} 

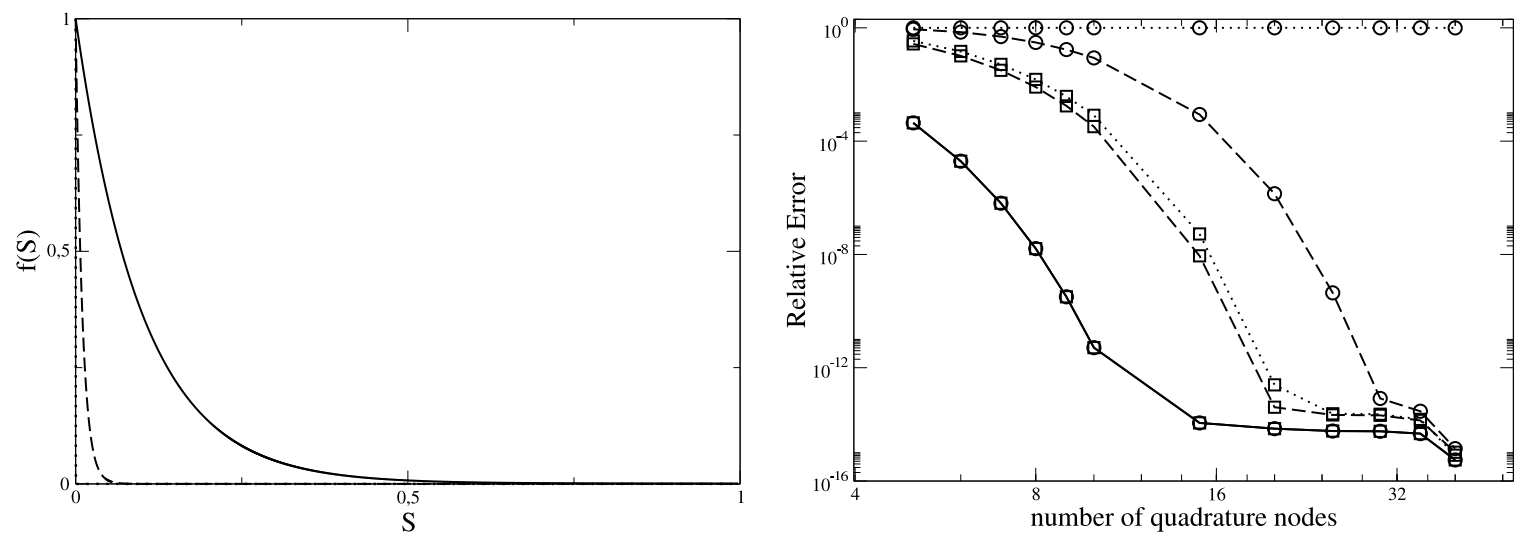

Figure 3: Adaptive integrals: (left) Function $f(S)=\exp (-K S)$ for $K=10^{1}$ (full line), $K=10^{2}$ (dashed line) and $K=10^{4}$ (dot-dashed line) and (right) relative error on the integrals with fixed (circle) and adaptive (square) supports.

we are close to a zone of the frontier where the uniquely defined NDF involves a lower number of variables. For example for canonical moments $p_{1} \in(0,1)$ and $p_{2}=0$ on the frontier, only 3 parameters are necessary to reconstruct the NDF, which ends to be the lower order representation. So the idea is that there is a transition zone in which we go from a situation where 4 parameters are needed (the interior of the moment space), to a situation where less parameters are needed (the frontier). We then propose to determine where to use less than four moments is effective in the sense that we reach the same level of accuracy without leading to unstable numerical methods. The introduced error has to be controlled, as only the frontier is uniquely determined by less moments. The main interest of such a strategy is that the computational cost and the convergence rate of the Newton solver may be notably reduced. Furthermore, the computational time of the EM being mainly dominated by cells where the NDF is close to the frontier of the moment space, one can adapt the accuracy on the frontier to control the computational time.

One can notice that each moment linearly depends on the canonical moment of the same order if lower order moments are fixed. So, with respect to $p_{k}$, and $M_{0}^{0}, \ldots, M_{0}^{k-1}$ being fixed, the moment $m_{k}$ is bounded between $M_{0}^{k^{m i n}}=\left.M_{0}^{k}\right|_{p_{k}=0}$ and $M_{0}^{k^{\max }}=\left.M_{0}^{k}\right|_{p_{k}=1}$. The distance $\delta M_{0}^{k}$ between minimum and maximum values normalized by the zeroth moment is then defined as:

$$
\delta M_{0}^{k}=\frac{\left.M_{0}^{k}\right|_{p_{k}=1}-\left.M_{0}^{k}\right|_{p_{k}=0}}{M_{0}^{0}} .
$$

So if $M_{0}^{3}$ is considered (here the highest order moment), $\delta M_{0}^{3}$ is:

$$
\delta M_{0}^{3}=p_{1} p_{2}\left(1-p_{1}\right)\left(1-p_{2}\right) .
$$

When $\delta M_{0}^{3}$ is close to 0 , the moment $M_{0}^{3}$ only weakly depends on $p_{3}$, i.e. the moment $M_{0}^{3}$ can be accurately reproduced with the first three moments only. The subset where it is sufficient to control the first three moments to achieve an accuracy $\epsilon_{3}=10^{-3}$ on $M_{0}^{3}$ is represented in Fig. 4. When the moment vector is closer to the frontier of the moment space than this limit, the number of controlled moments is reduced from 4 to $3^{5}$

The same procedure can be applied to reduce from 3 to 2 moments using the normalized distance for $M_{0}^{2}$ :

$$
\delta M_{0}^{2}=p_{1}\left(1-p_{1}\right),
$$

\footnotetext{
${ }^{5}$ Let us underline that this amount to reducing the dimensionality of the interior of the moment space in a neighborhood of its frontier, with a given tolerance on higher order moments, in such a way that the highest order moment(s) becomes a function of the lower order moments through the EM reconstruction of the lower order moments. Such an approximation of the higher order moments, considering the "thickness" of the moment space in such a neighborhood, provides an accurate value.
} 

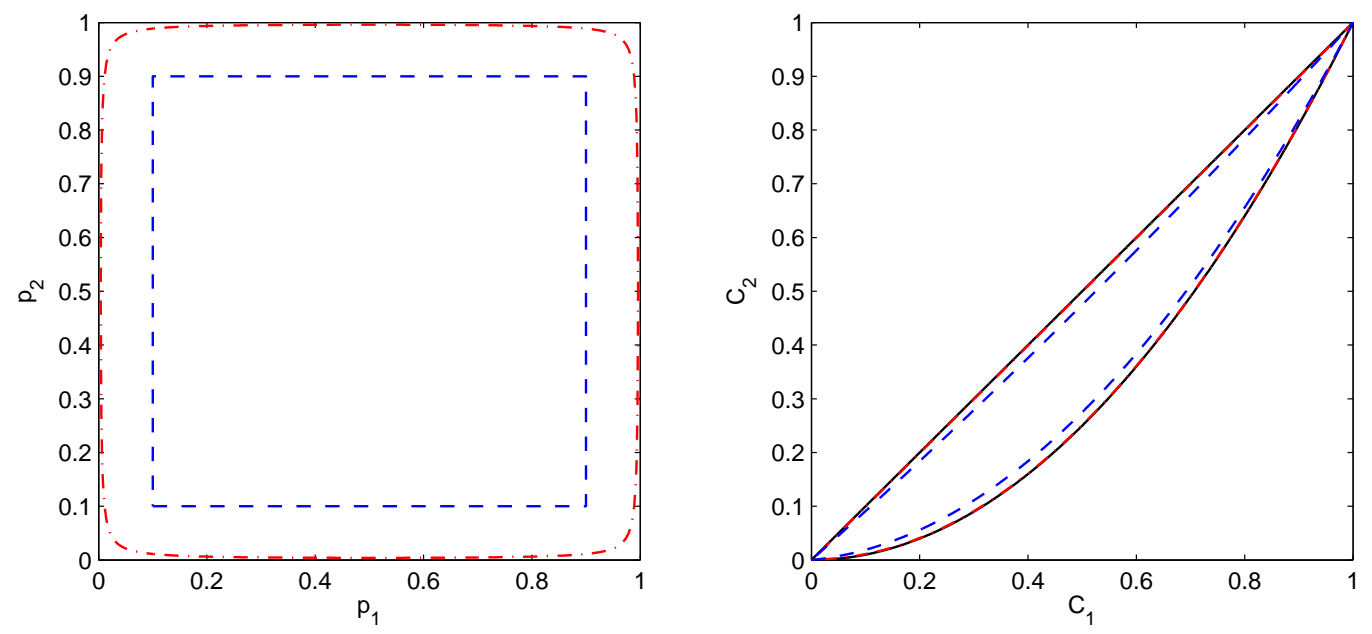

Figure 4: Subsets of the moment space where tabulation and reduction of controlled moments are used against $p_{1}$ and $p_{2}$ (left: canonical moment space, right: normalized moment space): limit within the tabulation is envisaged with a tolerance of $10^{-4}$ (blue dashed line) and limit within 3 size moments are sufficient to achieve a precision of $10^{-3}$ on $M_{0}^{3}$ (red dot-dashed line). The black line is the limit of the realizable moment space.

so that if $\delta M_{0}^{2}$ is close to zero the number of controlled moments can be reduced from 3 to 2 . This condition being more restrictive than the one on $\delta M_{0}^{3}$, fulfilling it will be sufficient to reduce from 4 to 2 the number of controlled moments. Once again, such a process can be extended to high order moments in a straightforward manner.

\subsection{Conclusions on the entropy maximization}

The adaptive support for integrals enables an accurate computation of the integrals of almost singular NDFs. With the knowledge of the canonical moments, we are now able to choose an optimal initial guess for the Newton solver by means of a tabulation. The number of moments used for the reconstruction can be adapted where less moments are needed. This adaptivity is limited to a subspace close to the frontier, and we are able to define accurately the error made by this reduction of the number of parameters.

Thus, the EM is now able to reproduce a large subset of the moment space, and is ready for highly segregative cases such as the crossflow (Fig. 1), where the drag force separates the droplets into a quasimonodisperse NDF, which corresponds to moment vectors living close to the frontier of the moment space. Let us underline that turbulence will naturally take the distribution away from the frontier of the moment space, as studied in [42].

\section{Accounting for size-velocity correlation: the CSVM method}

\subsection{Description of the problem}

The initial EMSM considers that all droplet sizes have the same velocity. In moderate to high Stokes flows, the velocity of each size would be different, as exhibited in Fig. 1 in the crossflow configuration, leading to the following expression for the NDF:

$$
f(t, x, v, S)=n(t, x, S) \delta(v-U(t, x, S))
$$

where $U$ depends on position and time, but also on droplet surface. To describe this velocity distribution, a reconstruction strategy is envisioned. The starting point is the same as the initial EMSM for the reconstruction of the size distribution: with a certain number of moments, we need to have an accurate enough description of the NDF. As we want to build size-velocity informations, size-velocity moments will be used 
so that the CSVM will use size moments to determine the size distribution $n(S)$ and size-velocity moments to determine $U(S)$. As the determination of $n(S)$ does not need $U(S)$, since size moments do not depend on $U(S)$, this first step of the EMSM is unchanged. However the velocity reconstruction depends on the size reconstruction. Three constraints are imposed for our reconstruction strategy:

- Constraint 1: droplets with a null size, i.e. a null relaxation time, follow the gas,

- Constraint 2: the presumed shape for $U(S)$ must not be monotonic, to reproduce relative velocity inversion that may occur in turbulent flow (due to the oscillating nature of the gas velocity),

- Constraint 3: the reconstruction strategy has to work in a Multifluid framework. It means that the method have to work with one size interval, but also with several size intervals coupled by droplet fluxes due to evaporation.

\subsection{Strategy for the velocity reconstruction}

Hereafter, the reconstruction strategy for one dimension is detailed. The application for other directions is straightforward, as no second order moments in velocity will be used $(i \leq 1)$ :

$$
M_{i}^{l}=\int_{0}^{1} S^{l} U(S)^{i} n(S) \mathrm{d} S .
$$

To evaluate the velocity of each size, a generic shape for $U(t, x, S)$ need to be presumed. Following constraint 1:

$$
U(t, x, S=0)=u_{g}
$$

To be able to represent all possible moment vectors, it is necessary that the reconstruction can describe the full velocity moment space. The following power reconstruction is suggested:

$$
U(t, x, S)=u_{g}+\sum_{k=1}^{N_{v}} A_{k} S^{\alpha_{k}}
$$

where $N_{v}$ is the number of size-velocity moments, $A_{k}$ are the parameters used to control the size-velocity moments, and $\alpha_{k}$ are user-determined with $\alpha_{1}<\alpha_{2}<\cdots<\alpha_{N_{v}}$. Considering size-velocity moments expressions, one can write:

$$
M_{1}^{l}=M_{0}^{l} u_{g}+\sum_{k=1}^{N_{v}} A_{k} \int_{0}^{1} S^{\alpha_{k}+l} n_{l}(S) \mathrm{d} S,
$$

which gives the linear system for the parameter vector $\mathcal{A}=\left(A_{1}, \ldots, A_{N_{v}}\right)^{T}$ :

$$
P \mathcal{A}=\mathcal{N}
$$

where:

$$
\begin{aligned}
P_{k l} & =\int_{0}^{1} S^{\alpha_{k}+l} n_{l}(S) \mathrm{d} S \\
N_{l} & =M_{1}^{l}-M_{0}^{l} u_{g}
\end{aligned}
$$

Considering Eq. (34), the only configuration for which this reconstruction will not be possible for a given moment vector corresponds to the zero determinant for matrix $P$. This condition is reached for a Dirac $\delta$-function for the size distribution $n(S)$. As the EM does not generate this type of distribution, our reconstruction strategy can reproduce any moment vector given by our method. Due to constraint $2, N_{v}$ must be greater or equal than to 2, to allow a change of sign for the derivative. Finally, constraint 3 is already satisfied, as the proposed strategy does not depend on the size interval, but directly on the moments. So it 
can be applied on any section, always keeping an information on the gas velocity, even for big droplets (it does not mean that the minimum size of each interval has the same velocity as the gas phase).

In [14], an exponential reconstruction is used, based on the analytical solution of the relaxation of droplets at constant gas velocity. Although this is a physical and relatively smooth reconstruction, with good asymptotic predictions, this shape is not able to reproduce all the moment space. Basically, in the case of an oscillating gas velocity, small droplets react fastly to the gas velocity variations, whereas big droplets have a bigger relaxation time. This may lead to different dynamics in the size interval: the sign of the relative velocity between the gas and the droplets may change in the size interval. This type of dynamics cannot be captured by monotonic functions with a zero size constraint, such as in [14] or [17].

\section{Numerical methods for the moment evolution}

\subsection{Evaporation and drag force}

We present a new strategy for the evolution of the NDF through evaporation and drag force. The method described in [15] is based on two main steps: the evaluation of the disappearance flux of droplet, and the evolution in size space. The strategy is here extended to the evolution in velocity space through drag force. First the derivation is shown for the $d^{2}$ law and Stokes drag $\left(R_{S}=\right.$ cst and $\left.\operatorname{St}(S)=K_{d} S\right)$. The size reconstruction of the CSVM enables the computation of the disappearance flux of droplets $F_{i}^{l}$ for each moment and for one time step $\Delta t$ :

$$
F_{i}^{l}=\int_{0}^{-R_{S} \Delta t} U(S)^{i} S^{l} n_{l}(S) \mathrm{d} S,
$$

corresponding to the part of the distribution which reaches the zero size during one time step. The moment are then corrected:

$$
\widetilde{M}_{i}^{l}=M_{i}^{l}-F_{i}^{l} .
$$

The evolution in phase space is determined by a CQMOM-like quadrature approach [40] :

$$
\begin{aligned}
\widetilde{M}_{i}^{l} & =\sum_{k=1}^{2} w_{k} S_{k}^{l} U_{k}^{i}, \\
\frac{\mathrm{d} U_{k}}{\mathrm{~d} t} & =-\frac{1}{\mathrm{St}}\left(U_{k}-u_{g}\right)=-\frac{1}{K_{d} S_{k}}\left(U_{k}-u_{g}\right), \\
\frac{\mathrm{d} S_{k}}{\mathrm{~d} t} & =R_{S} .
\end{aligned}
$$

The size and velocity after one time step is:

$$
\begin{aligned}
S_{k}(t+\Delta t) & =S_{k}(t)+R_{S} \Delta t \\
U_{k}(t+\Delta t) & =\left(U_{k}(t)-u_{g}(t)\right)\left(\frac{S_{k}(t)+R_{S} \Delta t}{S_{k}(t)}\right)^{\overline{K_{d} R_{S}}}+u_{g}(t) .
\end{aligned}
$$

and the updated moments are:

$$
M_{i}^{l}(t+\Delta t)=\sum_{k=1}^{2} w_{k}(t) S_{k}(t+\Delta t)^{l} U_{k}(t+\Delta t)^{i} .
$$

To account for more complex law, the strategy is slightly modified, the two differences lying on the flux determination and the ODE system that is needed to be solved. Considering $R_{S}(S)$ and $\operatorname{St}(S)$, the evaluation of the disappearance flux of droplets is now:

$$
F_{i}^{l}=\int_{0}^{S_{l i m}(t)} U(S)^{i} S^{l} n_{l}(S) \mathrm{d} S
$$


where $S_{\text {lim }}$ is obtained by solving the non-linear system backward in time:

$$
\left\{\begin{array}{l}
\frac{\mathrm{d} S_{\text {lim }}}{\mathrm{d} t}=R_{S}\left(S_{\text {lim }}\right), \\
S_{\text {lim }}(t+\Delta t)=0,
\end{array}\right.
$$

which means that $S_{l i m}$ is the biggest size that reaches the zero size during one timestep. Using Eq. (43), the corrected moments are computed and the same quadrature is performed, as with simple laws. Then, the obtained system of ODE is solved:

$$
\left\{\begin{array}{l}
\frac{\mathrm{d} U_{k}}{\mathrm{~d} t}=-\frac{1}{\operatorname{St}(S)}\left(U_{k}-u_{g}\right), \\
\frac{\mathrm{d} S_{k}}{\mathrm{~d} t}=R_{S}\left(S_{k}\right),
\end{array}\right.
$$

Using an ODE solver, the updated moments are computed in the same manner as with simple laws. Furthermore, this strategy works in a Multifluid approach, since fluxes can be calculated for each section in a finite volume manner.

\subsection{Flux splitting kinetic scheme}

The physical space evolution is surely the most important part of the size-velocity correlation. As seen in previous section, the constant velocity assumption, even if it leads to higher errors, does not lead to a completely different moment evolution in the velocity phase space. But in physical space, as shown by Fig. 1, the dynamics will be totally different. The moment equation for the transport in physical space in one dimension is:

$$
\frac{\partial \mathcal{M}}{\partial t}+\frac{\partial \mathcal{F}(\mathcal{M})}{\partial x}=0,
$$

where $\mathcal{M}=\left(M_{0}^{0}, M_{0}^{1}, M_{0}^{2}, M_{0}^{3}, M_{1}^{0}, M_{1}^{1}\right)^{T}$ is the moment vector and $\mathcal{F}(\mathcal{M})=\left(M_{1}^{0}, M_{1}^{1}, M_{1}^{2}, M_{1}^{3}, M_{2}^{0}, M_{2}^{1}\right)^{T}$ is the flux vector. For a constant velocity distribution, the fluxes are linear functions of the moments. In this case, a first order finite-volume scheme preserves the moment space, as it is positive definite and the scheme reconstruction generates realizable moments. The second-order scheme that preserves the moment space is much more difficult to design, and this issue raised in the literature [43] had been finally tackled in [26]. In the case of a continuous non-constant velocity, fluxes are now a complex function of the moments, and designing a specific scheme becomes necessary.

The moment equation is related to the following one dimensional kinetic equation:

$$
\frac{\partial f}{\partial t}+v \frac{\partial f}{\partial x}=0
$$

where $f(t, x, v, S)=n(t, x, S) \delta(v-U(t, x, S))$. Integrating over velocities but not over sizes, the following infinite system for $S=[0,1]$ is found:

$$
\begin{aligned}
\frac{\partial n(t, x, S)}{\partial t}+\frac{\partial n(t, x, S) U(t, x, S)}{\partial x} & =0, \\
\frac{\partial n(t, x, S) U(t, x, S)}{\partial t}+\frac{\partial n(t, x, S) U(t, x, S)^{2}}{\partial x} & =0 .
\end{aligned}
$$

To solve this system, a first order upwind finite volume scheme is used. Defining the cell values at each time step ${ }^{6}$ :

$$
\begin{aligned}
n_{j}^{n}(S) & =\frac{1}{\Delta x} \int_{x_{j-1 / 2}}^{x_{j+1 / 2}} n\left(\Delta t^{n}, x, S\right) \mathrm{d} x \\
U_{j}^{n}(S) & =\frac{1}{\Delta x} \int_{x_{j-1 / 2}}^{x_{j+1 / 2}} U\left(\Delta t^{n}, x, S\right) \mathrm{d} x
\end{aligned}
$$

\footnotetext{
${ }^{6}$ Here exponent $n$ qualifies the time step, and not a variable at the power $n$.
} 
The moment update is:

$$
\begin{array}{r}
n_{j}^{n+1}=n_{j}^{n}-\frac{\Delta t}{\Delta x}\left(F_{j+1 / 2}-F_{j-1 / 2}\right), \\
n_{j}^{n+1} U_{j}^{n+1}=n_{j}^{n} U_{j}^{n}-\frac{\Delta t}{\Delta x}\left(G_{j+1 / 2}-G_{j-1 / 2}\right),
\end{array}
$$

where:

$$
\begin{aligned}
F_{j+1 / 2} & =\min \left(0, U_{j+1}^{n}\right) n_{j+1}^{n}+\max \left(0, U_{j}^{n}\right) n_{j}^{n}, \\
G_{j+1 / 2} & =\min \left(0, U_{j+1}^{n}\right) n_{j+1}^{n} U_{j+1}^{n}+\max \left(0, U_{j}^{n}\right) n_{j}^{n} U_{j}^{n} .
\end{aligned}
$$

By integrating over size, the following scheme is obtained:

$$
\mathcal{M}_{j}^{n+1}=\mathcal{M}_{j}^{n}-\frac{\Delta t}{\Delta x}\left(\mathcal{F}_{j+1 / 2}-\mathcal{F}_{j-1 / 2}\right)
$$

where the fluxes are splitted into positive and negative components:

$$
\mathcal{F}_{j+1 / 2}=\int_{0}^{1} \mathcal{S}\left(\min \left(0, U_{j+1}^{n}\right) n_{j+1} \mathcal{U}_{j+1}^{n}+\max \left(0, U_{j}^{n}\right) n_{j} \mathcal{U}_{j}^{n}\right) \mathrm{d} S=\mathcal{F}_{j+1}^{-}+\mathcal{F}_{j}^{+},
$$

where $\mathcal{S}=\left[1, S, S^{2}, S^{3}, 1, S\right]^{T}$ and $\mathcal{U}_{j}^{n}=\left[1,1,1,1, U_{j}^{n}, U_{j}^{n}\right]^{T}$. Using the size and velocity reconstructions, fluxes in Eq. (56) can be computed for every moments using the adaptive integration method proposed for the EM. To ensure the stability of the scheme, a CFL condition is imposed on the timestep which is also based on the velocity reconstruction:

$$
\Delta t<\mathrm{CFL} \frac{\Delta x}{\max _{S}(|U(S)|)}
$$

The two main interests of the resulting numerical scheme is that it reproduces the behavior at the kinetic level and is proven realizable. The originality is that, comparing to classical kinetic schemes which have to handle the dependence in size of the maximum velocity to integrate the fluxes exactly in time, the proposed scheme totally disconnects the maximum velocity from size (by taking the maximum over the whole size spectrum), and then allows an easy closure of the scheme. This scheme is first order in time and space only, which will be not sufficient for real applications. A second order scheme is proposed in Appendix A, but is not used here, as a first order scheme is sufficient to highlight the main features of size-velocity correlations.

\section{Evaluation on simple $0 \mathrm{D}$ and $1 \mathrm{D}$ configurations}

The CSVM is evaluated on simple $0 \mathrm{D}$ and $1 \mathrm{D}$ configurations. These test cases are set to assess the accuracy of the reconstruction strategy as well as the ability of the moment evolution methods to capture the main features of size-velocity correlations:

- The reconstruction strategy is validated, by evaluating the reconstruction error for both NDF and velocity reconstructions (Sec. 6.1),

- The moment evolution algorithms in phase space (drag force and evaporation) are evaluated on 0D cases with increasing complexity (Sec. 6.2),

- The convection scheme is finally investigated. Its ability to reproduce the impact of size-velocity correlations is validated through two 1D segregative cases, whereas the robustness is assessed on a 1D $\delta$-shock-generating case (Sec. 6.3). 

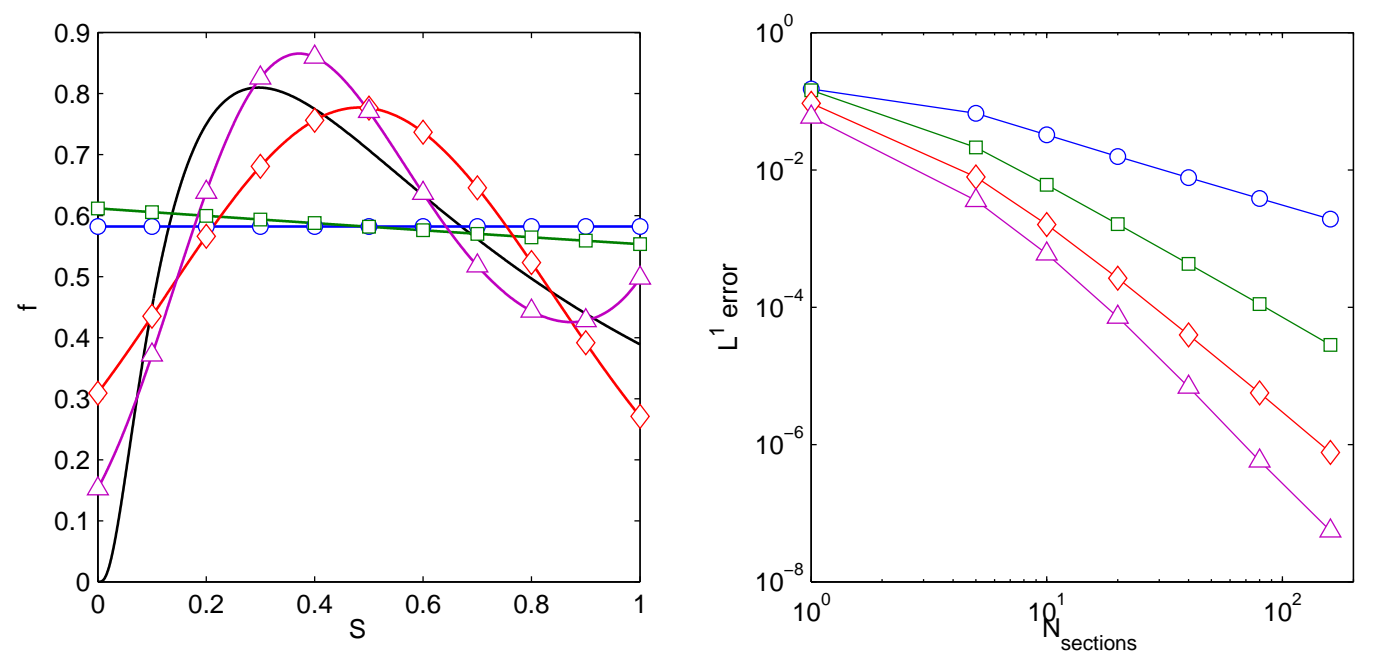

Figure 5: Entropy Maximization: reconstructed NDF for one section and $N=0, \ldots, 3$ (left) and $\mathrm{L}^{1}$ reconstruction error against the number of sections (right) for $N=0$ (blue circles ), 1 (green squares), 2 (red diamonds) and 3 (magenta triangles).

\subsection{Evaluation of the reconstruction strategy}

\subsubsection{NDF reconstruction}

The quality of the NDF reconstruction was already assessed in [15]. The new information that we provide concerns a accurate determination of the order of the method with respect to the number of controlled moments, by varying the number of sections. Moreover, we emphasize on an important feature of the size reconstruction: to adapt the precision of the method, one can either modify the number of controlled moments in a section, or modify the number of sections. It leads that the resulting reconstruction is highly adaptive, as it has two degree of freedom concerning the description of the NDF.

The initial NDF is a log-normal distribution on the compact support $[0,1]$ :

$$
f_{0}(S)=\frac{1}{S \sigma \sqrt{2 \pi}} \exp \left(-\frac{(\ln (S)-\ln (\mu))^{2}}{\sigma^{2}}\right)
$$

with $\sigma=1$ and $\mu=0.81$. In Fig. 5 , the reconstructed NDF and the $\mathrm{L}^{1}$ error against the number of sections are plotted, for different number of moments. The results show the increasing precision with the number of moments, as well as with the number of sections. With 4 moments, the method is proven to be of fourth order. We also demonstrate the direct link between the number of moments and the order of the method. It also demonstrates that the user can reach the same accuracy either by increasing the number of sections or by increasing the number of moments per section. In this work, we stay with one section, as our aim is to evaluate the method which uses the minimal number of moments.

\subsubsection{Velocity reconstruction}

To evaluate the reconstruction, the first $0 \mathrm{D}$ analytical test consists in an initial size-velocity distribution which evolves because of the drag force imposed by a constant gas velocity. The initial size and velocity distributions are constant so that $n(S, t)=1$ and $U(S, t=0)=U^{0}$. For each size, this case is solution of the ODE:

$$
\frac{\mathrm{d} U(S, t)}{\mathrm{d} t}=-\frac{U(S, t)-u_{g}}{\mathrm{St}(S)},
$$

SO:

$$
U(S, t)=u_{g}+\left(U^{0}-u_{g}\right) \exp \left(-\frac{t}{\operatorname{St}(S)}\right)
$$




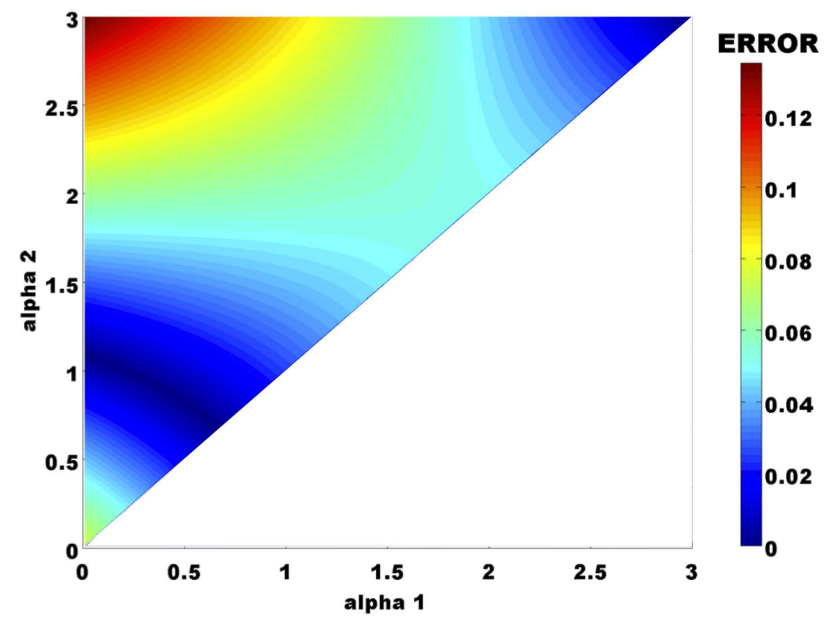

Figure 6: CSVM reconstruction error for the 0D test case at constant gas velocity for $t=1$ against $\alpha_{1}$ and $\alpha_{2}$. For $\alpha_{2}<\alpha_{1}$ the error is not plotted. For $\alpha_{1}=\alpha_{2}$, the reconstruction is undefined.

The moment evolution against time is:

$$
\begin{aligned}
& M_{1}^{l}(t)=\int_{0}^{1} S^{l} U(S, t) n(S) \mathrm{d} S=\frac{u_{g}}{l+1}+\left(U^{0}-u_{g}\right) \int_{0}^{1} S^{l} \exp \left(-\frac{t}{\operatorname{St}(S)}\right) \mathrm{d} S, \\
& M_{1}^{0}(t)=u_{g}+\frac{t}{K_{d}}\left(U^{0}-u_{g}\right) \Gamma_{i n c}\left(-1, \frac{t}{K_{d}}\right), \\
& M_{1}^{1}(t)=\frac{u_{g}}{2}+\frac{t^{2}}{K_{d}^{2}}\left(U^{0}-u_{g}\right) \Gamma_{i n c}\left(-2, \frac{t}{K_{d}}\right),
\end{aligned}
$$

where $\Gamma_{\text {inc }}(a, z)=\int_{z}^{\infty} x^{a-1} \exp (-x) \mathrm{d} x$ is the upper incomplete gamma function and $\operatorname{St}(S)=K_{d} S$, which corresponds to Stokes drag.

In Fig. 6, the influence of the power $\alpha_{1}$ and $\alpha_{2}$ on the absolute reconstruction error in $L^{\infty}$ norm is shown for $t=1$. Two optimal zones are exhibited, the first one with high powers nearly identical, and the second one with one power close to 1 and the second one lower than 1 . In practice, $\alpha_{1}=0.5$ and $\alpha_{2}=1$ will be used. Results are plotted in Fig. 7 (left). For a two parameter reconstruction, it proves to be efficient except for a constant velocity distribution, which artificially breaks the assumption $U(S=0)=u_{g}$.

The second analytical test case has a variable gas velocity $u_{g}=\alpha \cos (\beta t)$. It aims at representing the velocity profile inside a vortex, and so the effect of a turbulent structure. The solution of Eq. (59) is then:

$$
U(S, t)=\frac{\alpha(\beta \operatorname{St}(S) \sin (\beta t)+\cos (\beta t))}{\beta^{2} \operatorname{St}(S)^{2}+1}+\frac{\left[U^{0} \beta^{2} \operatorname{St}(S)^{2}+\left(U^{0}-\alpha\right)\right] \exp \left(-\frac{t}{\operatorname{St}(S)}\right)}{\beta^{2} \operatorname{St}(S)^{2}+1} .
$$

Results are plotted in Fig. 7 (right). Again the reconstruction is satisfactory with only two moments and so two parameters, and proves to be able to reproduce distributions with an inversion of the gas relative velocity, which would be typical of turbulent flows with a wide spectrum of droplet sizes. Furthermore, as a relative velocity inversion occurs, an exponential function would not be able to reproduce such a distribution.

So we are able to reconstruct the droplet number density as well as the velocity for each size. Now, we need to predict the evolution of this NDF through evaporation, drag force and convection.

\subsection{Moment evolution: OD test cases}

Two 0D test cases are used to evaluate the ability of the CSVM to account for both drag and evaporation. For all cases, the initial liquid velocity distribution is constant and set to $U(S)=1$. The initial size 

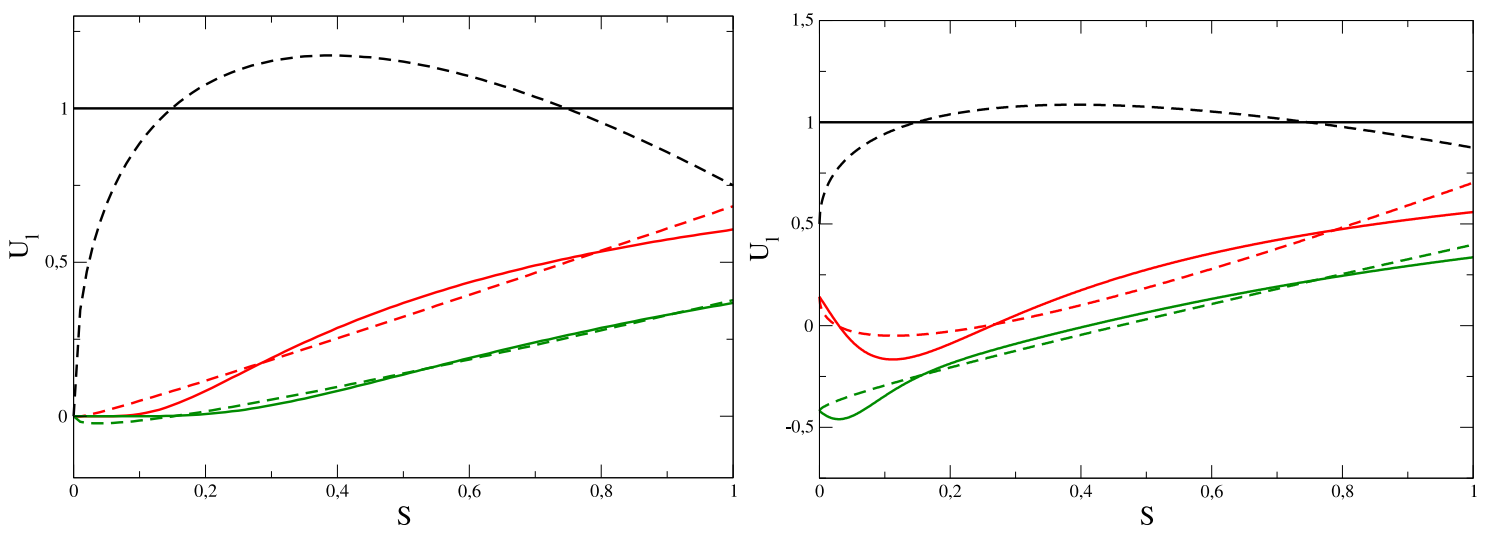

Figure 7: 0D analytical test case with constant (left) and sinusoidal (right) gas velocity: velocity distribution (solid lines) and its reconstruction by CSVM (dashed lines).

distribution is a normal distribution $n(S)=1 /(\sqrt{2 \pi} \sigma) \exp \left(-(S-\mu) / 2 \sigma^{2}\right)$ with $\sigma=0.4$ and $\mu=0.6$. The Stokes number at $S=1$ is set to 1 . Then the test cases are defined by two parameters: the gas velocity as a function of time, $u_{g}(t)$, and the evaporation rate $R_{S}$. The Lagrangian reference is computed using a tracking of $10^{6}$ particles randomly chosen to fullfill the normal distribution $n(S)$. Eulerian quantities for the reference solution are obtained by computing the moment of the Lagrangian particles.

The combined effect of drag force and evaporation is evaluated. Evaporation is the first motivation of the EMSM, and its accuracy has already been demonstrated in [15]. The novelty of this work is the ability to achieve a good accuracy on size-velocity moments. Here we consider $R_{S}=-1$ and $u_{g}=0.5 \cos (10 t)$. The evolution of moments as a function of time for Lagrangian, CSVM and EMSM are plotted in Fig. 8. For size moments, the results are the same for CSVM and EMSM, as the size reconstruction is the same. For size-velocity moments, results are better for the CSVM. This result is confirmed by Fig. 9, on which the error for size and size-velocity moments is plotted. For the CSVM, the error is under $3 \%$ where it reaches $10 \%$ without velocity reconstruction, and this with only one additional moment.

To investigate the ability of the method to capture a more complex dynamic, this test case will consider no evaporation $\left(R_{S}=0\right)$ and a variable gas velocity with three modes, plotted in Fig. 10:

$$
u_{g}(t)= \begin{cases}\frac{1}{2} \cos (4 \pi t), & t \leq 1, \\ \cos (\pi t), & 1<t \leq 5, \\ \frac{1}{4} \cos (8 \pi t), & t>5\end{cases}
$$

These three modes are expected to mimic what can be seen by particles going through several vortices with different properties. In Fig. 11, the evolution of size-velocity moments against time for CSVM and EMSM. The improvement of the velocity reconstruction is obvious, the error on $M_{1}^{1}$ being undistinguished with velocity reconstruction. These results are confirmed again by the error in Fig. 12, which stays under $2 \%$ with reconstruction and reaches more than $10 \%$ without.

Finally, the CSVM is able to capture the phase space evolution due to evaporation and drag force of a dispersed phase, by using only one additional moment comparing to the EMSM, which cannot capture the evolution of size-velocity moments.

\section{3. $1 D$ convection cases}

The coupling between size-velocity correlations and convection can produce a high segregation with respect to droplet sizes. In the subsequent test cases, three aspects of the CSVM are highlighted: 

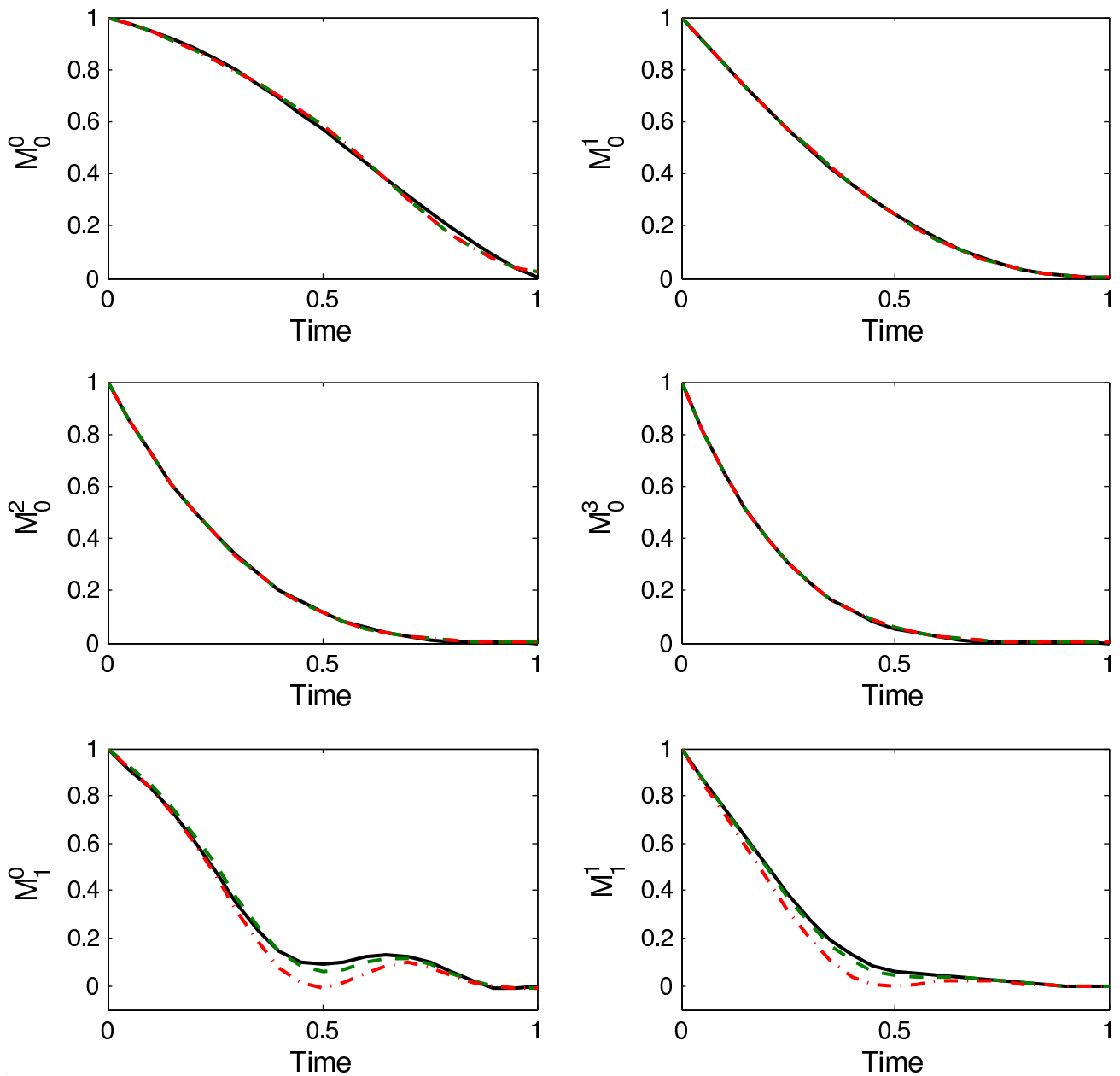

Figure 8: Evolution of resolved moments: Lagrangian reference (solid line), CSVM (dashed line), and EMSM (dot-dashed line).
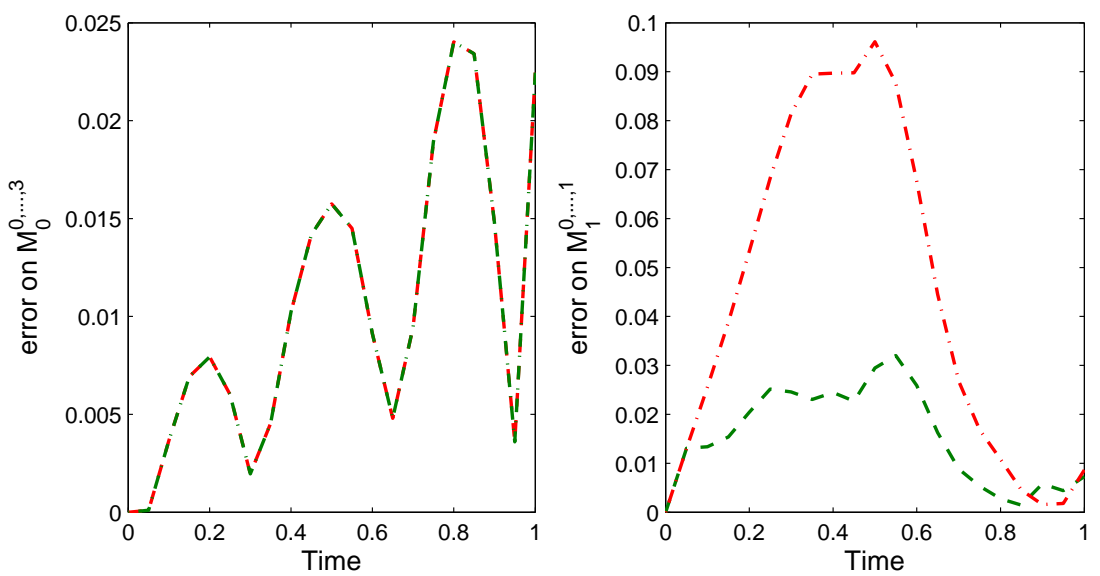

Figure 9: Evolution of the maximal error on size (left) and size-velocity moment (right) vectors comparing to the Lagrangian reference: CSVM (dashed line), and EMSM (dot-dashed line) for size moments (left) and size-velocity moments (right). 


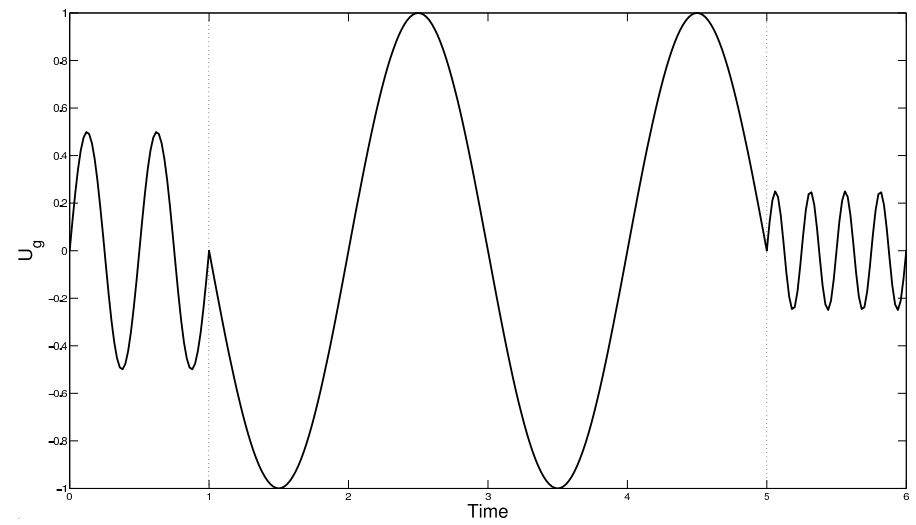

Figure 10: Evolution of the gas velocity against time.
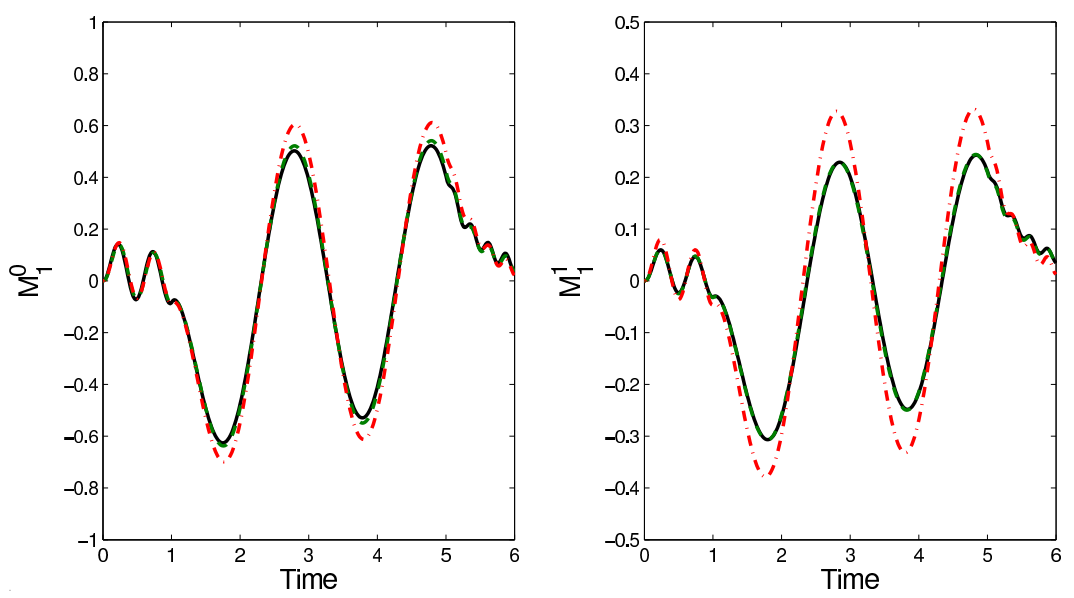

Figure 11: Evolution of resolved moments: Lagrangian reference (solid line), CSVM (dashed line), and EMSM (dot-dashed line).

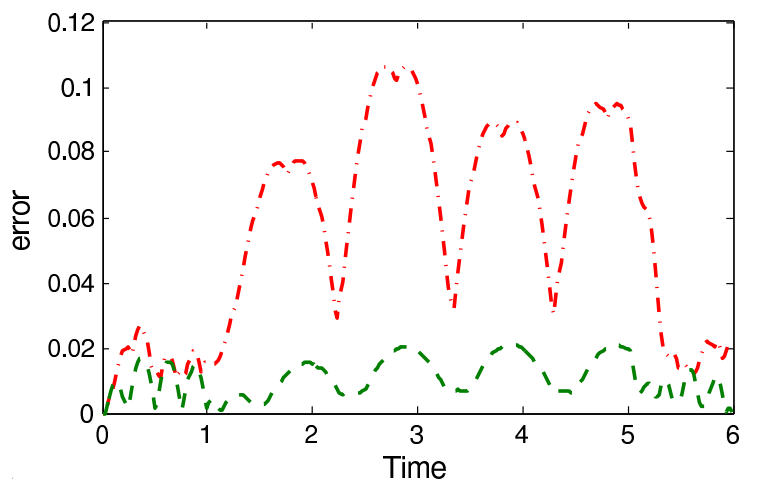

Figure 12: Evolution of the maximal error on size-velocity moments $M_{1}^{0}$ and $M_{1}^{1}$ comparing to the Lagrangian reference: CSVM (dashed line), and EMSM (dot-dashed line). 
- The ability of the convection scheme to reproduce size-conditioned dynamics (without drag force), and its order of accuracy (Sec. 6.3.1),

- The influence of the size reconstruction on the spatial convergence rate of the CSVM (Sec. 6.3.2),

- The accuracy and the robustness of the method when droplets trajectory crossings occur, leading to the generation of $\delta$-shocks in the monokinetic limit (Sec. 6.3.3).

\subsubsection{Segregation due to convection}

The first test case corresponds to a gaussian spatial distribution for total number density, whereas the NDF is constant in size, and the velocity is linear in size:

$$
\begin{aligned}
n(t=0, x, S) & =\exp \left(-\frac{\left(x-x_{c}\right)^{2}}{\sigma^{2}}\right), \\
U(t=0, x, S) & =U_{g}+\left(U_{\text {max }}-U_{g}\right) S .
\end{aligned}
$$

Here $x_{c}=0.2, \sigma=0.05, U_{g}=0, U_{\max }=1$. Such a test case is expected to produce a high segregation, as each size has its proper trajectory. For each size, the solution consists in the linear advection of the initial solution: $n(t, x, S)=n(0, x-U(t=0, x, S) t, S)$. Integrating over the whole size space, the analytical solution for $M_{0}^{0}$ at time $t=0.6$ is:

$$
M_{0}^{0}(x, t)=\frac{\sigma \sqrt{\pi}}{2 t\left(U_{1}-U_{g}\right)}\left[\operatorname{erf}\left(\frac{U_{1} t+x_{c}-x}{\sigma}\right)-\operatorname{erf}\left(\frac{U_{g} t+x_{c}-x}{\sigma}\right)\right] .
$$

where $U_{1}=U(S=1)$. Results are plotted in Fig. 13. The total droplet number is spread by the velocity distribution, and the agreement between analytical and CSVM solutions is satisfactory. The order of the scheme is 1 , and the method converges to the analytical solution.

\subsubsection{Convergence in physical and size spaces}

The previous test case is interesting to qualify the order of the method, but as it generates smooth distributions that can be accurately captured by the CSVM, one may want to assess the accuracy of the method when non-smooth NDF are generated. The test case now consists in a constant number density in size and space $n(x, S)=1$, but with a velocity distribution which has the following triangular hat distribution in space:

$$
U(x, S)= \begin{cases}0 & \text { if } x<0.2 \\ S(x-0.2) / 0.2 & \text { if } 0.2 \leq x<0.4 \\ S(0.6-x) / 0.2 & \text { if } 0.4 \leq x<0.6 \\ 0 & \text { if } x \geq 0.6\end{cases}
$$

The initial solution in velocity and the analytical solution of number density for three sizes are plotted in Fig. 14. This test case generates rectangular-hat-shaped distributions in size space, which are hardly reproduced by any smooth reconstructions ${ }^{7}$. Here we investigate the sectional aspect of the proposed method: the convergence rate is governed by the accuracy in both physical and size spaces.

In Fig. 15, the CSVM solutions with 1000 cells for 1 and 8 sections are compared to the analytical solution, and the error against the number of sections for different number of sections is plotted. It can be noticed that even if the size discretization is fine, the 1-section solution is not yet converged, whereas the 8 -section solution reproduces the analytical solution accurately. This issue is confirmed by the convergence rate against the number of cells, which reach first order (the limit imposed by the spatial scheme) with at least 4 sections for a coarse discretization, and 8 sections for a fine one. A main outcome of this test case is that the precision of the method is limited by the precision of each solver. Here, even using a fine resolution for the physical space, the error is still high because the description of the size space is not sufficiently accurate. Increasing the precision in size space permits to recover a satisfactory precision in physical space. It clearly demonstrates the link between the kinetic description of the NDF, and the resulting moment evolution.

\footnotetext{
${ }^{7}$ We recall that the EM builds the smoother distribution for a given moment set.
} 

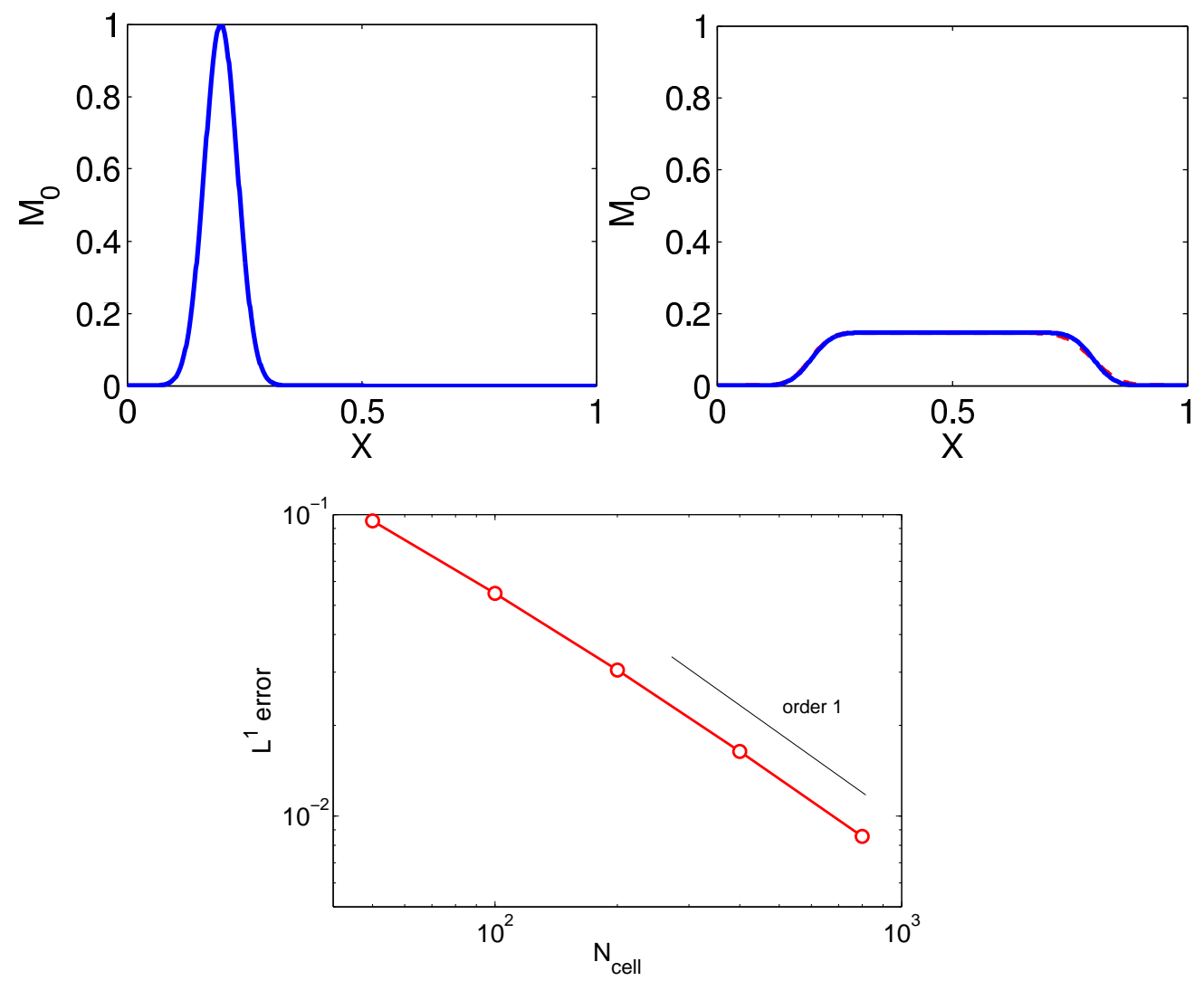

Figure 13: 1D test case: initial total droplet number distribution (upper left), analytical solution at $t=0.6$ (upper right, full line), CSVM solution at $t=0.6$ for 400 cells (upper right, dashed line), absolute error against cell number (down).
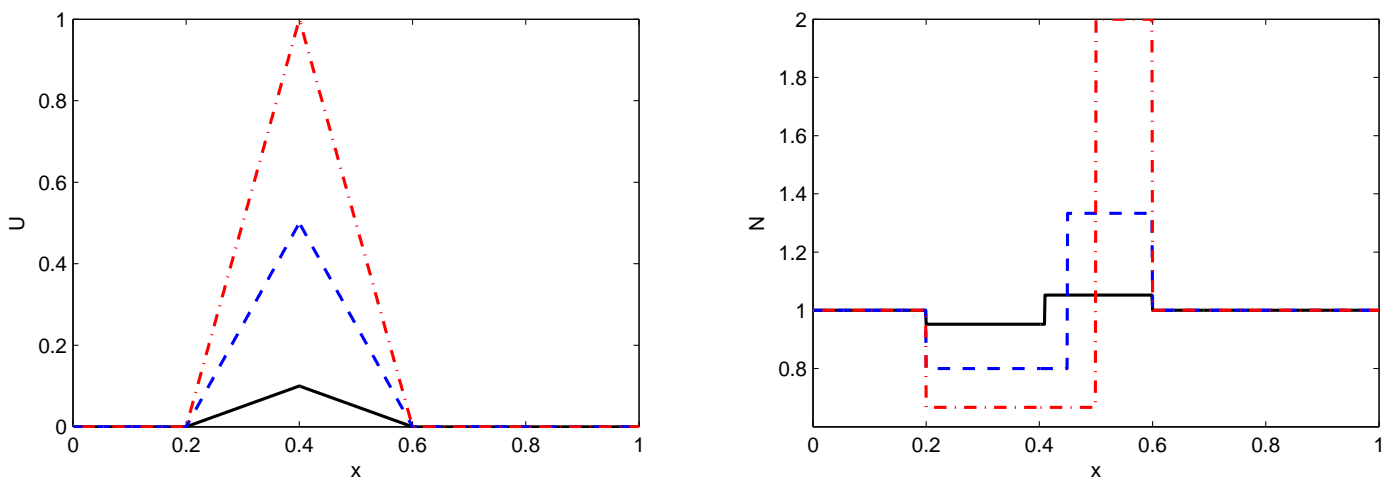

Figure 14: Triangular hat: initial velocity (left) and final number density at $t=0.1$ (right) for droplet sizes $S=0.1$ (full black), $S=0.5$ (dashed blue) and $S=1.0$ (dot-dashed red) 

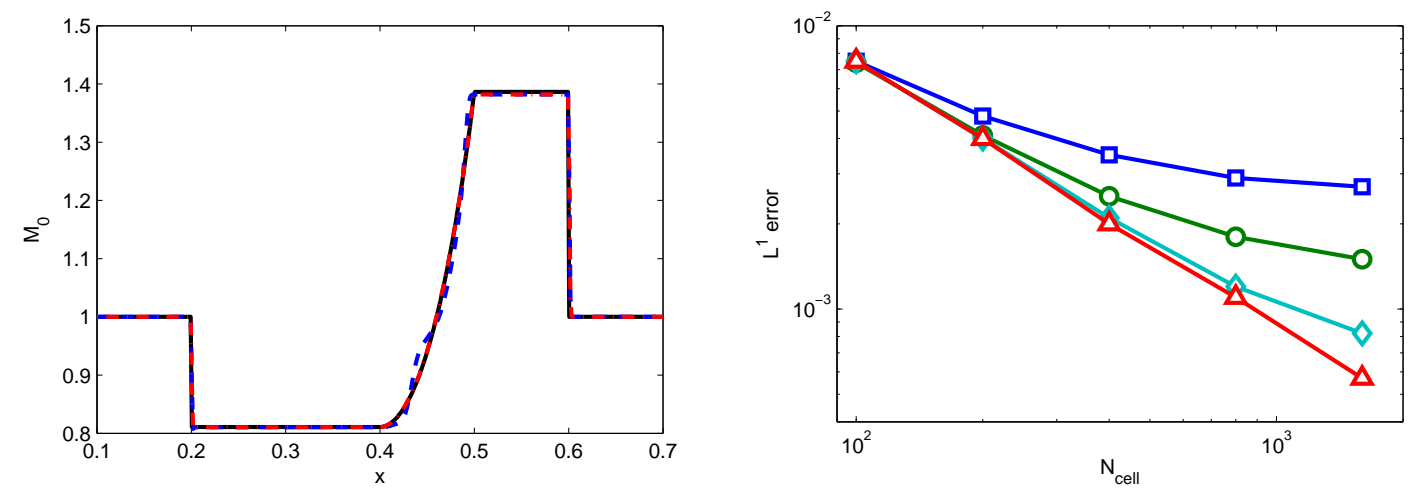

Figure 15: Triangular hat: (left) analytical solutions at $t=0.1$ (full black), CSVM results with 1 (dashed blue ) and 8 (dot-dashed red) sections, (right) $\mathrm{L}^{1}$ error against the number of cells for 1, 2, 4 and 8 sections

\subsubsection{Ability to capture $\delta$-shocks}

Even if the CSVM is able to capture the velocity associated with each size, it cannot capture the trajectory crossing for each size. Because the underlying semi-kinetic system is weakly hyperbolic, this will generate $\delta$-shocks ${ }^{8}$. To evaluate the ability of the method to capture accurately $\delta$-shock for each size, a 1D test case which is motivated by the Taylor-Green vortices is investigated. This configuration was further analyzed in [44, 45].

In a $1 \mathrm{D}$ domain $[-\pi, \pi]$, a steady gas velocity field is set with $u_{g}=-\sin (x)$. Droplets are distributed uniformly at $t=0$ with a null velocity and a constant droplet number density in size in the interval $\left[0,20 \mathrm{St}_{c}\right]$ where $\mathrm{St}_{c}=1 / 8 \pi$. In this test case all trajectories meet at $x=0$, generating a central $\delta$-shock, which is fed by all sizes progressively.

In Fig. 16 (left and center), droplet number densities are plotted at different times. It confirms the ability of the scheme to capture $\delta$-shocks accurately. In Fig. 16 (right), the comparison of the Multifluid with 1000 sections and the CSVM confirms the abilitv of the method to canture size-conditionned dvnamics. even in the case
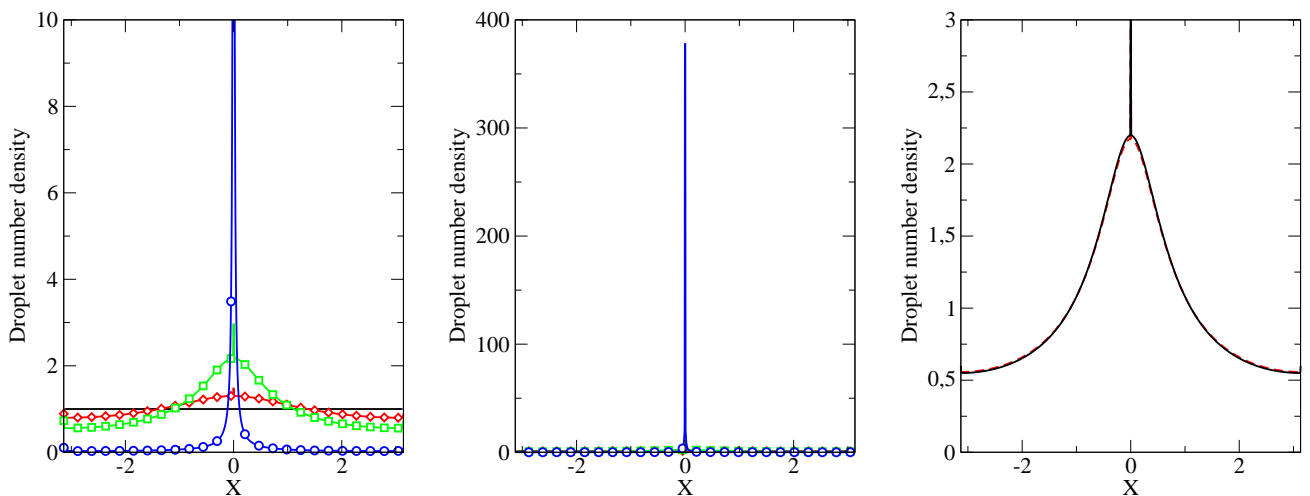

Figure 16: $\delta$-shock generation: droplet number density zoomed on the initial solution (left) and at full scale (center) at time $t=0$ (black line), $t=0.5$ (red line with diamonds), $t=1.0$ (green line with squares) and $t=5.0$ (blue line with circles). Droplet number density at time $t=1.0$ for CSVM (dashed red line) and Multifluid with 1000 sections (full black line) (right).

\footnotetext{
${ }^{8}$ In the limit of infinite Knudsen number, these $\delta$-shocks are unphysical. This issue has been tackled in [2] using high order moments in velocity, in the context of EMSM. The same approach can be envisaged in the context of the CSVM, but is out of the scope of this paper.
} 


\section{Application to complex cases}

Three 2D complex cases are investigated: the crossflow, Taylor-Green vortices and a weakly turbulent free jet. The first one consists in the injection of particles in the normal direction of a constant gas flow, which leads to a high size segregation and exhibits a steady solution. The second one consists in four contra-rotating vortices in a periodic domain. This case also leads to a high size segregation, but is more complicated as the gas velocity is not uniform. Those two test cases are set to evaluate the ability of the method to capture a limiting case, i.e. when all the moment sets in the domain tend to the frontier of the moment space. In practical applications, the NDF tends to smooth size distributions because of the turbulent mixing for example (see [42] where polydispersion is exactly generated from a monodisperse spray through evaporation and turbulent mixing). The last test case is closer to pratical applications. it consists in the injection of particles into a weakly turbulent free jet. As our analysis will be focused on the dynamics of a polydisperse cloud of droplets, these test cases are non-evaporating.

\subsection{Crossflow}

The crossflow generates monodisperse distributions due to drag force size-conditioned segregation. As monodisperse distributions are at the frontier of the moment space, it is an interesting case to confirm the ability of the EM to capture such distributions. Moreover considering that every size has its proper velocity and trajectory, it is also a good case for evaluating the transport scheme.

The size of the computational domain is 2 in $x$ direction and 1 in $y$ direction. The injection is made between $X_{L}=0.1$ and $X_{R}=0.3$, at a constant velocity $V_{0}=1$ and $U_{0}=0$, with a constant number distribution $n(t, x, S)=1$. The gas flow is uniform with $U_{g}=1$ and $V_{g}=0$ and the Stokes number of the droplets is $\mathrm{St}=K_{d} S$ with $K_{d}=1$. The semi-analytical solution is described in Appendix B.

In Fig. 17, the droplet number density is shown for the Multifluid approach with 10 and 40 sections, and the CSVM with 4 moments in size and 2 moments in size-velocity. The Multifluid approach considers only one Stokes number for each section, so each section will have only one dynamics, even if the section covers a wide spectrum of Stokes numbers. The effect of such a size discretization is strong with 10 sections (30 moments, 10 in size, $2 \times 10$ in velocity), with 10 distinct trajectories. This effect becomes less pronounced with 40 sections (120 moments). With the CSVM, as the whole spectrum of Stokes number in the distribution is considered, a continuous number density distribution is obtained. And this is done with only 8 moments.

In Fig. 18, Multifluid approach with 10 and 40 sections, CSVM and analytical solution for the number density distribution and mean surface against $Y$ are compared at the right outlet of the domain. The highly oscillatory behavior of the 10 sections Multifluid solution is exhibited. The 40 sections Multifluid solution is in good agreement with the analytical solution. The CSVM captures a continuous distribution, but exhibits a large scale oscillation. In fact the results are encouraging for a 8 moments solution (to be compared with the 30 and 120 moments for Multifluid solution). The quality of the dynamical structure of the solution is confirmed by the mean size. All the methods captures relatively well the size distribution, with the worse results for the 10 section Multifluid and better results for the 40 section Multifluid. This test case also demonstrates the ability and the robustness of the CSVM to capture high segregation effects with a limited number of moments.

\subsection{Taylor Green vortices}

The Taylor Green vortices case is more complex than the Crossflow, as the gas velocity is not uniform. Furthermore, we focus on the transient regime. The segregation effect is strong, small droplets being captured by the vortices, whereas big droplets can move from a vortex to another. The computational domain is $[0,1]^{2}$ and periodic in each direction. The gas velocity is:

$$
\begin{aligned}
& U_{g}(X, Y)=\quad \cos (2 \pi X) \sin (2 \pi Y), \\
& V_{g}(X, Y)=-\sin (2 \pi X) \cos (2 \pi Y) .
\end{aligned}
$$

Droplets with a Stokes number higher than the critical Stokes number $\mathrm{St}_{c}=1 / 8 \pi$ can exhibit trajectory crossings, and thus a monokinetic Eulerian approach (one velocity per size in this work) can generate $\delta$-shock as the system is weakly hyperbolic [44, 45]. 

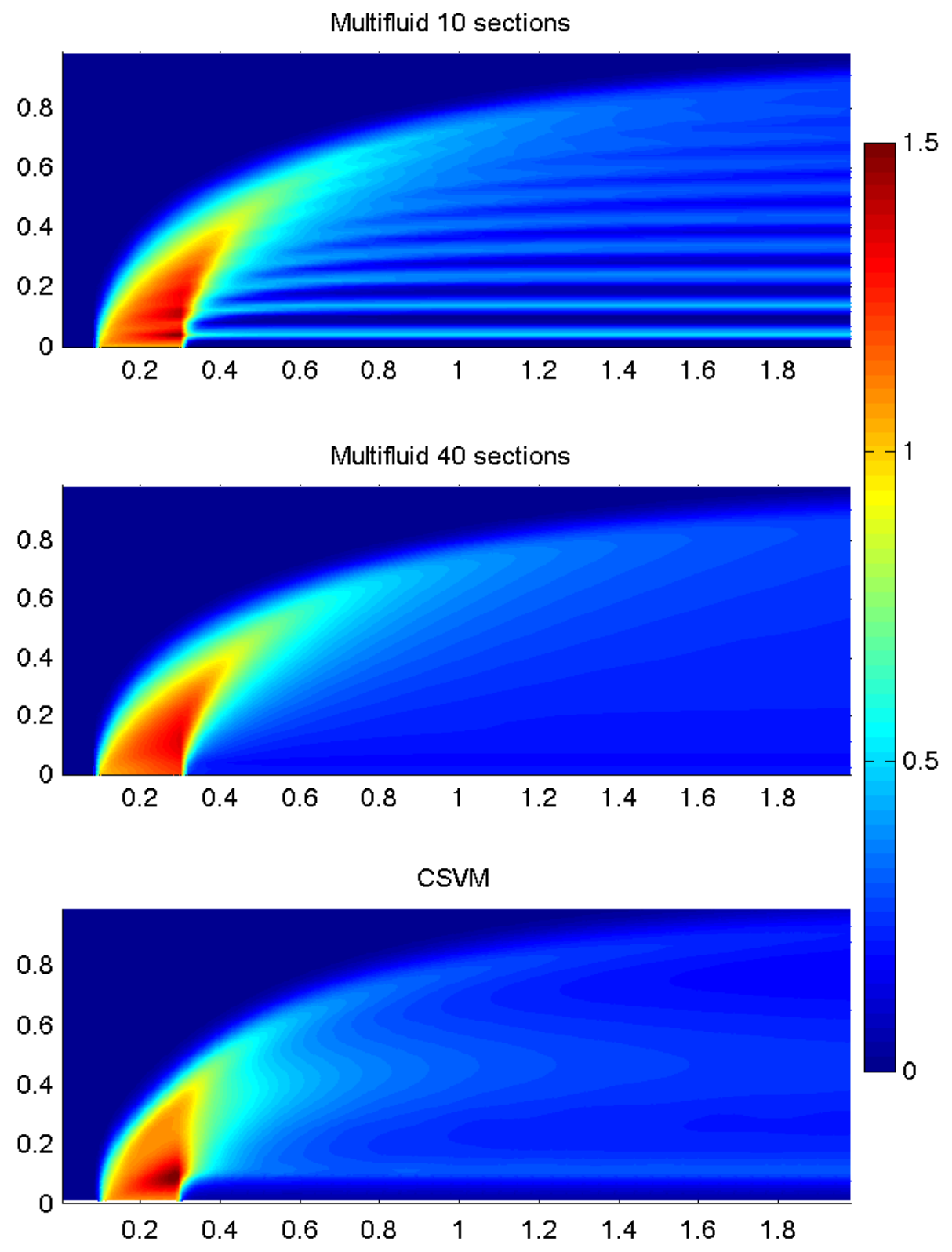

Figure 17: 2D Crossflow: droplet number density for the Multifluid approach with 10 sections (up) and 40 sections (center), and for the CSVM (down). 

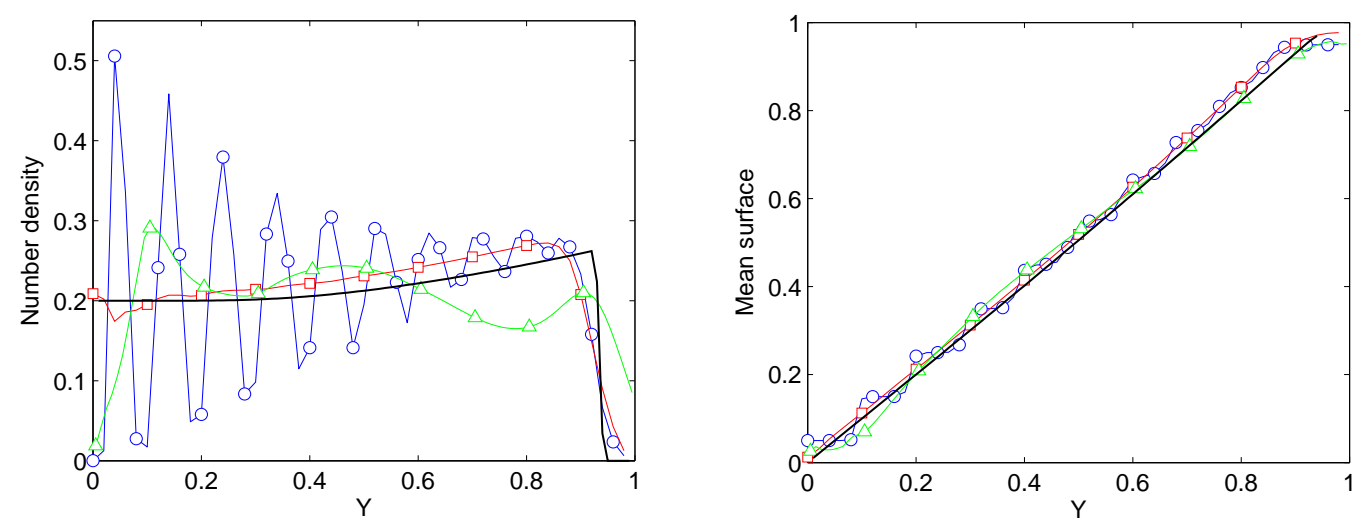

Figure 18: 2D Crossflow: droplet number density (left) and mean surface (right) at $X=2$ against $Y$ for the Multifluid approach with 10 sections (blue circles) and with 40 sections (red squares), the CSVM (green triangles) and the analytical solution (black line).

The initial number density distribution is :

$$
\begin{aligned}
n(x, y, t, S) & =\frac{\sin (\pi W R)}{\pi W R}, \\
R & =\sqrt{\left(x-x_{c}\right)^{2}+\left(y-y_{c}\right)^{2}},
\end{aligned}
$$

where $W=\sqrt{10}$ and $\left[x_{c}, y_{c}\right]=[0.275,0.825]$. The size interval is $\left[0,20 \mathrm{St}_{c}\right]$ where $\mathrm{St}_{c}=1 / 8 \pi$. The initial droplet velocity is equal to the gas velocity. Since no analytical solution can easily be obtained, a Lagrangian reference solution is used. A number of $10^{8}$ particles is used, in order to achieve a satisfactory statistical convergence. The Lagrangian system of equations is solved using an implicit first order scheme (see Appendix C), with a time step of $\Delta t=10^{-3}$.

The droplet number density (Fig. 19) and the mean surface (Fig. 20) for EMSM, 40 section Multifluid, Lagrangian, and CSVM are shown at time $t=1$. The EMSM solution shows the effect of having one velocity for the whole size distribution. Comparisons between the Lagrangian reference solution, the 40 sections Multifluid and the CSVM show a satisfactory agreement. For the mean surface, the results of the CSVM are satisfactory compared to the Lagrangian reference, and confirm the great accuracy of the method to capture the size-conditionned dynamics. The Multifluid also obtains satisfactory results, even if some oscillations appear due to the combined effect of vacuum zones between sections and numerical diffusion. 

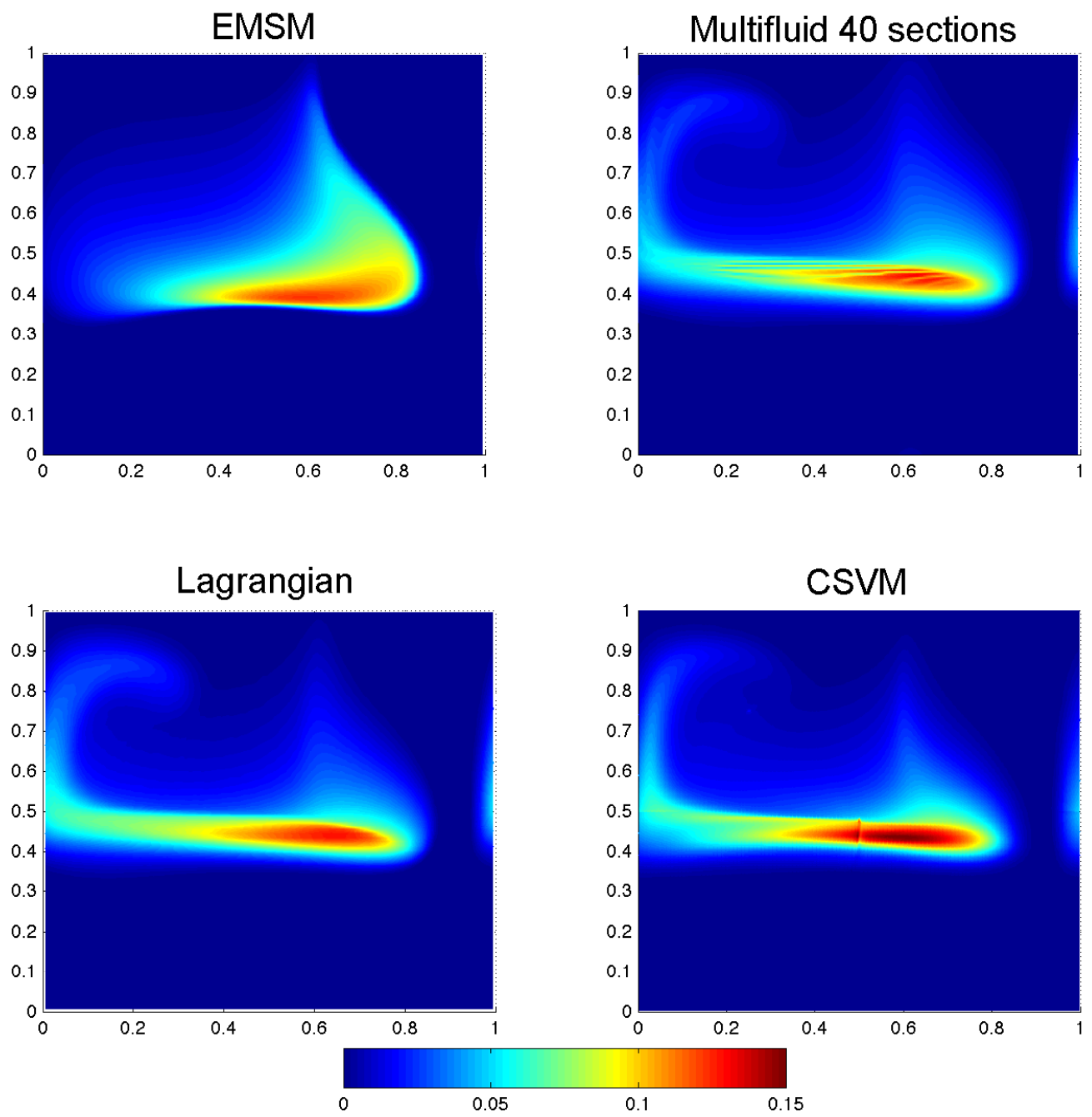

Figure 19: Taylor-Green vortices: droplet number density for the EMSM approach (upper left) and with 40 sections (upper right), the Lagrangian tracking with $10^{8}$ particles, and the CSVM. 

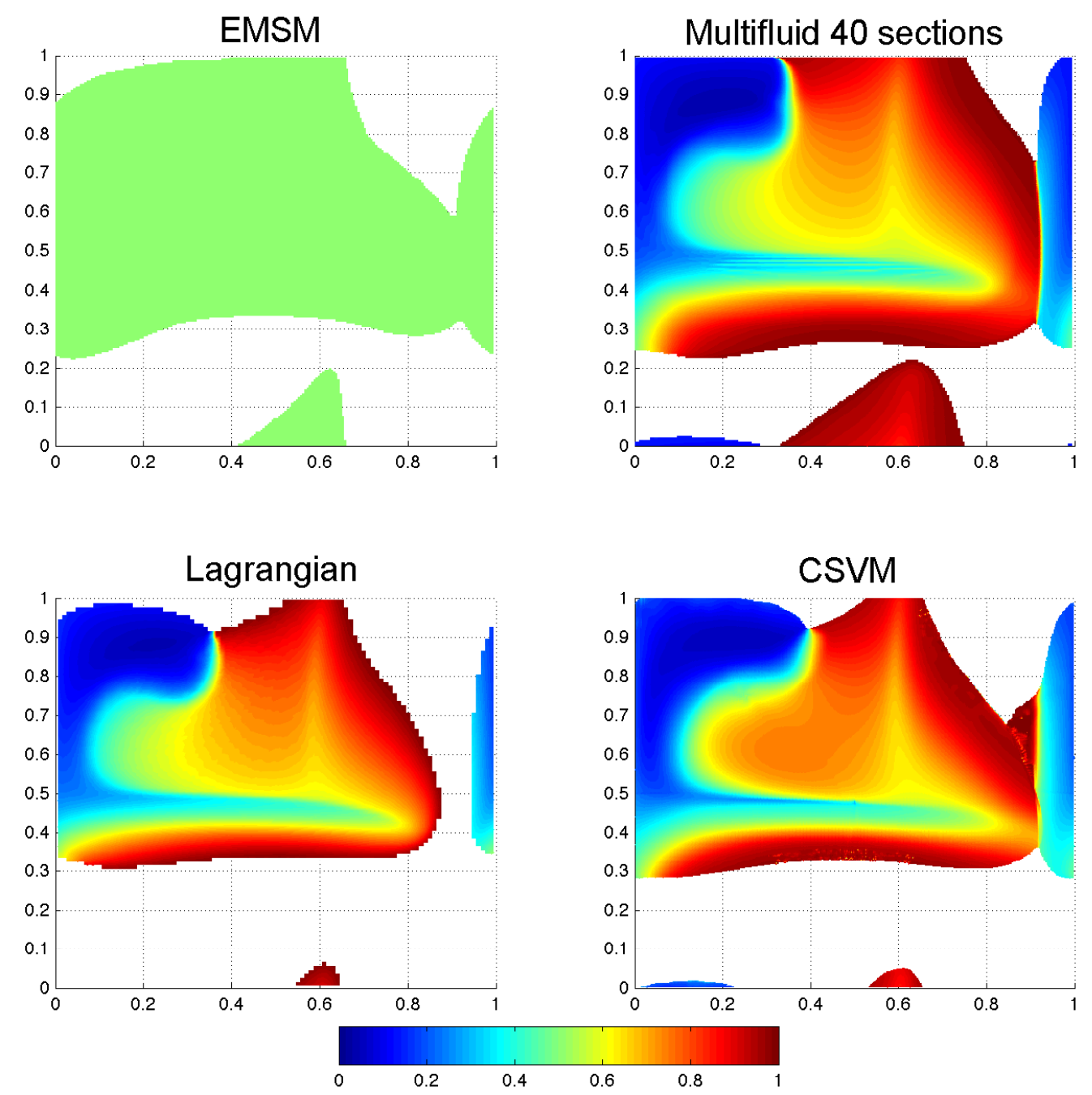

Figure 20: Taylor-Green vortices: mean surface for the EMSM approach (upper left) and with 40 sections (upper right), the Lagrangian tracking with $10^{8}$ particles, and the CSVM. 


\subsection{Weakly turbulent Free jet}

In this last test case, we consider an unsteady weakly turbulent free jet originally introduced in the Ph.D. Thesis of Hicham Meftah at CORIA [46]. It is generated using the ASPHODELE solver, developed at CORIA by Julien Reveillon and collaborators [47, 48], which uses a low Mach solver for the gas phase. Kah et al. [26] showed the ability of the EMSM to reproduce this case in evaporating conditions, but for a range of low Stokes numbers where the differential size-conditioned dynamics has a limited impact on the global spray dynamics. In the present case, in order to assess the accuracy and potential of the method, we rather study a non-evaporating case with a much larger range of Stokes number where size-conditioned differential dynamics effects are important.

The free jet Reynolds number based on $U_{0}, \nu_{0}$ and $L_{0}$ is 1000. For illustration purpose, dimensional quantities are given, the solver using non-dimensional quantities. $U_{0}=1 \mathrm{~m} / \mathrm{s}$ is the injection velocity, $\nu_{0}=1.5 \times 10^{-3} \mathrm{~m}^{2} / \mathrm{s}$ the gas viscosity, and $L_{0}=1.5 \mathrm{~cm}$ the jet width. To destabilize the jet, turbulence is injected using the Klein method with $10 \%$ fluctuations so that $U_{0}^{\prime}=0.1 \mathrm{~m} / \mathrm{s}$ [49]. The vorticity field is presented in Fig. 21. The Stokes number relative to the gas fluctuation time scale $\tau_{g}=L_{0} / U_{0}^{\prime}$ of the injected particles ranges from $\mathrm{St}=0$ to $\mathrm{St}=6.66$.

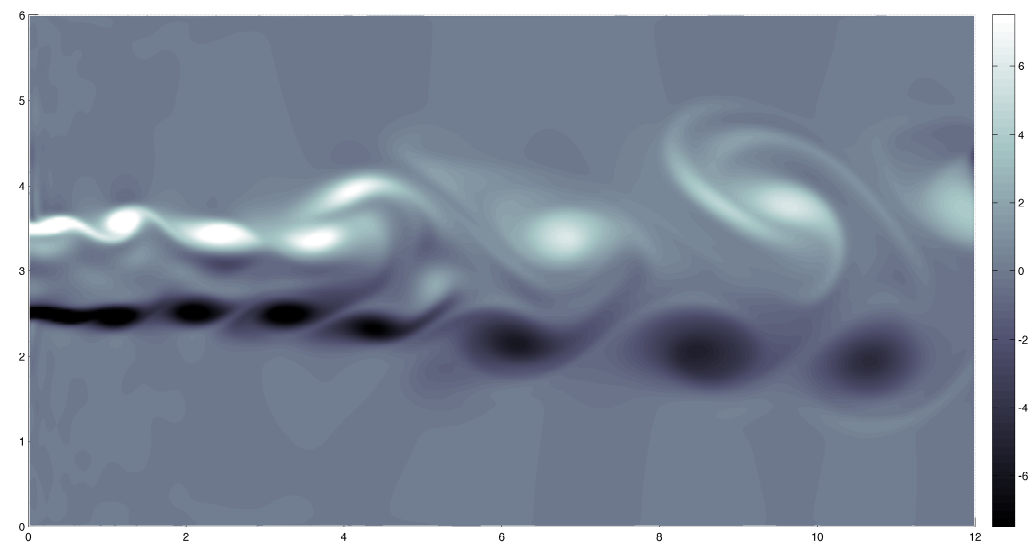

Figure 21: Free jet: vorticity field for the gas phase at time $t=20 \mathrm{~s}$.

Figure 22 and Fig. 23 show the number density and the mean surface for the EMSM and ten section Multifluid approaches, the Lagrangian method and the CSVM. All methods capture relatively well the number density spatial distribution, even if Eulerian results are more diffusive than the Lagrangian one.

In Figs. 24-25, the number densities for small droplets $(S=[0.0,0.1])$ and big droplets $(S=[0.9,1.0])$ are shown. Now, whereas the EMSM captures the same repartition for small and big droplets, the Multifluid approach and the CSVM are able to reproduce the differential dynamics for each size intervals: the big droplets are ejected from the large vortices of the free jet, whereas small droplets stay into them [50].

Those results are confirmed by the mean surface plotted in Fig. 23. The Lagrangian result shows the high size segregation induced by the vortices. The 10 section Multifluid approach and the CSVM show a good qualitative agreement with the reference solution, the numerical diffusion due to the first order convection schemes tending to diffuse the thin structures appearing in the Lagrangian simulation ${ }^{9}$.

It is now proven that the CSVM method provides an accurate description of the number density as well as size-velocity correlations of a polydisperse spray with a large range of Stokes number, successfully coping with the singularities at the frontier of the moment space where the dynamics tend to converge due to the size-segregation capabilities of the chosen gaseous flow fields.

\footnotetext{
${ }^{9}$ Once again, for the sake of fair comparisons, we have used a first order scheme for all Eulerian simulations.
} 

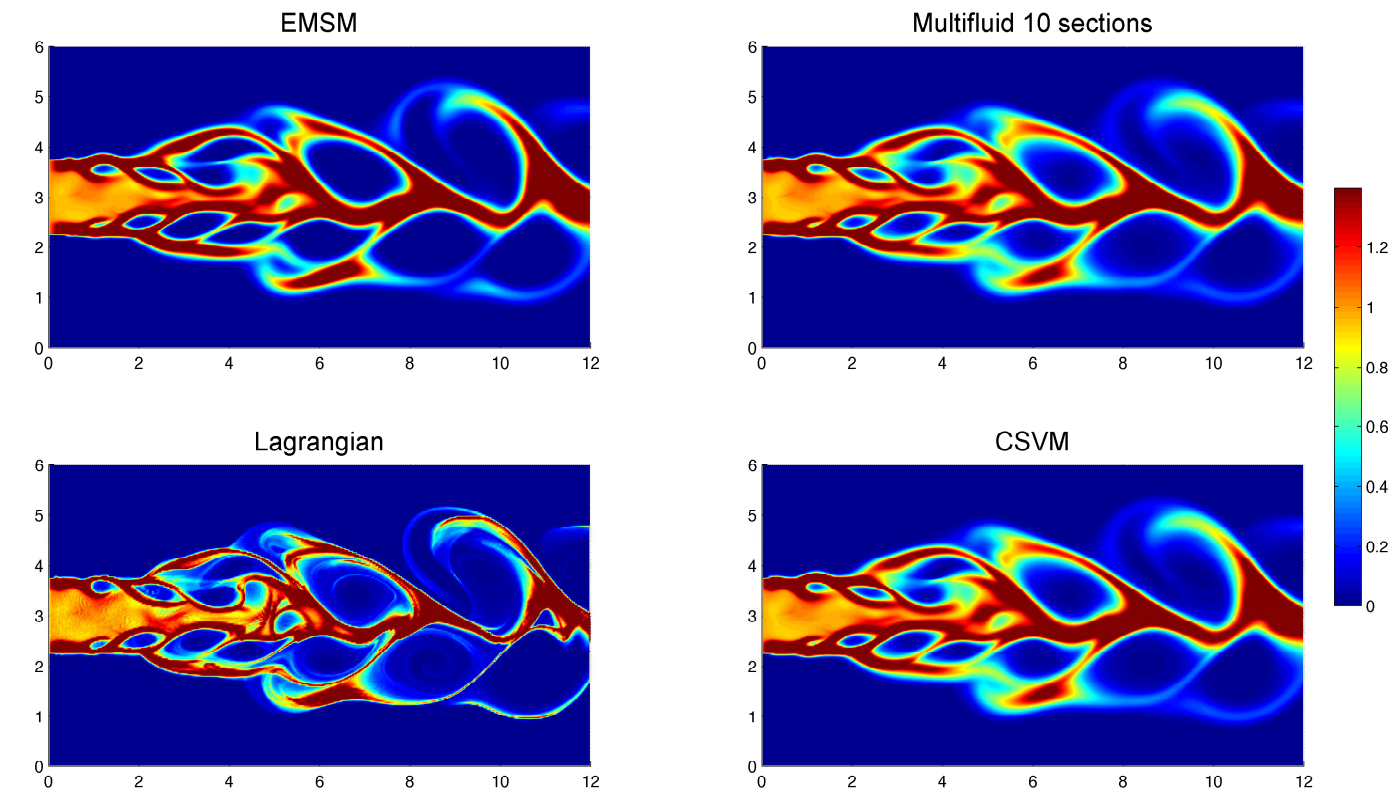

Figure 22: Free jet: droplet number density for the EMSM approach with one section (upper left) and with Multifluid 10 sections (upper right), the Lagrangian tracking with $10^{8}$ particles, and the CSVM.
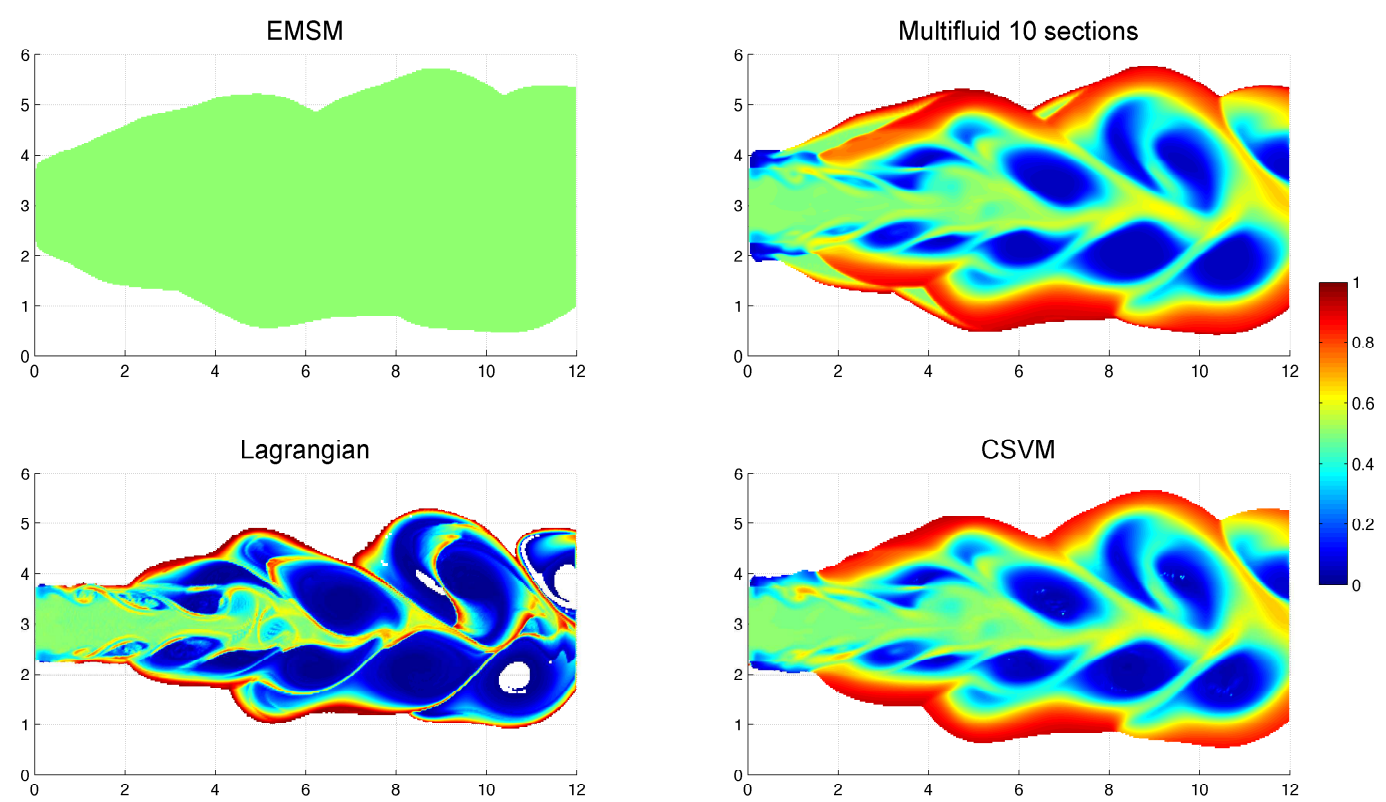

Figure 23: Free jet: mean surface for the EMSM approach with one section (upper left) and with Multifluid 10 sections (upper right), the Lagrangian tracking with $10^{8}$ particles, and the CSVM. 

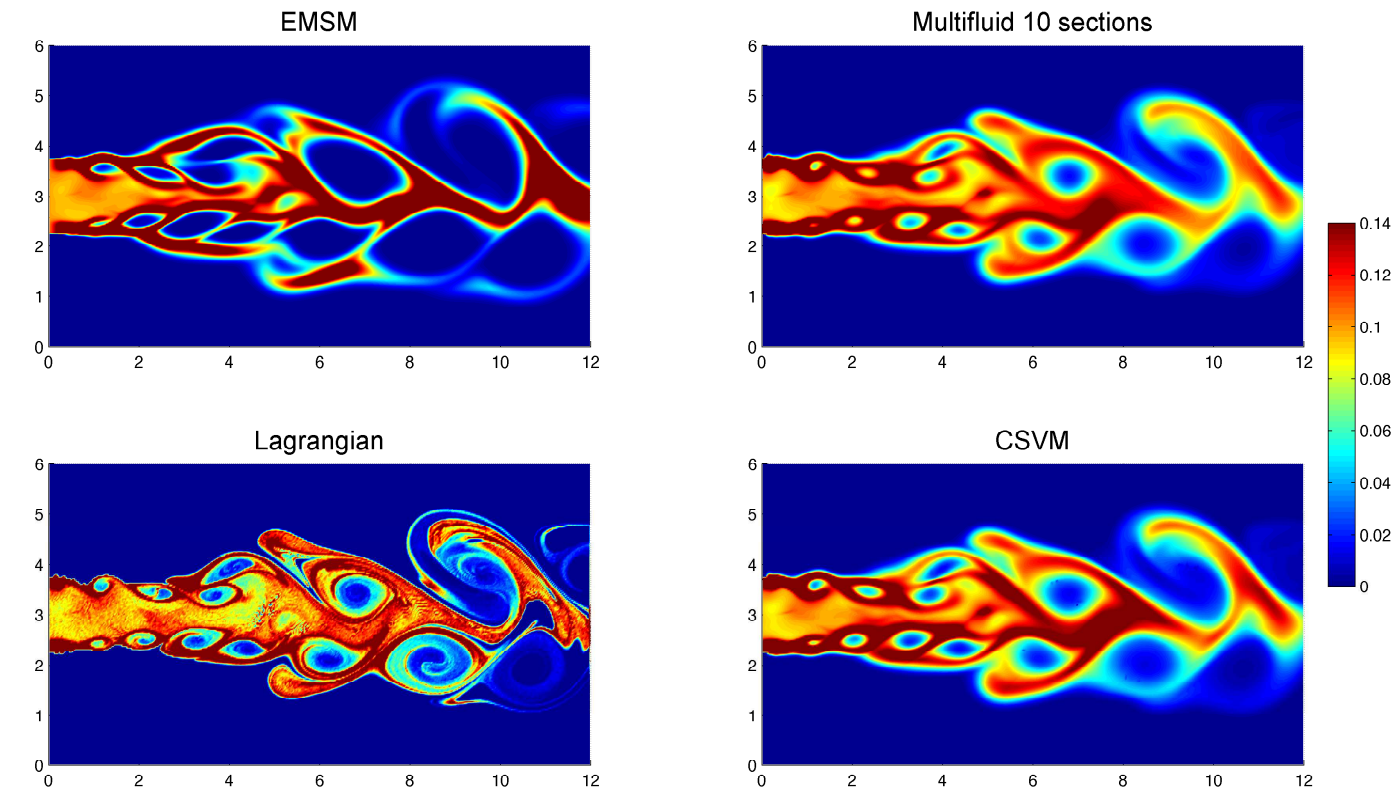

Figure 24: Free jet: droplet number density in $S=[0.0,0.1]$ for the EMSM approach with one section (upper left) and with Multifluid 10 sections (upper right), the Lagrangian tracking with $10^{8}$ particles, and the CSVM.
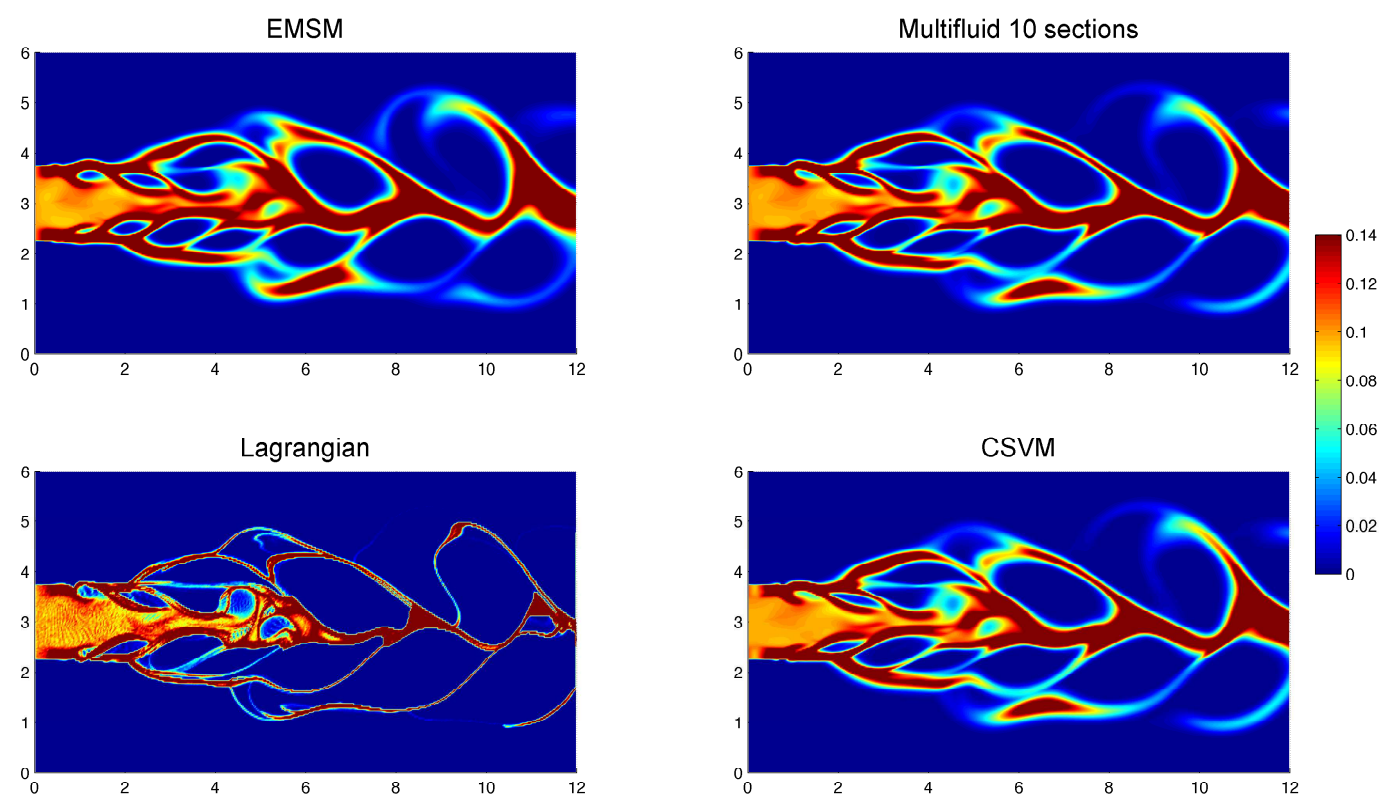

Figure 25: Free jet: droplet number density in $S=[0.9,1.0]$ for the EMSM approach with one section (upper left) and with Multifluid 10 sections (upper right), the Lagrangian tracking with $10^{8}$ particles, and the CSVM. 


\subsection{Computational Time}

In the laminar configurations we have chosen, the potential of the method has been highlighted and will lead to large memory storage savings in the case of 3D cases. In terms of computational cost, even if the configurations chosen in the present study are not favorable (since for example there is no subgrid turbulent mixing which will take the moment vector further away from the frontier of the moment space), the CSVM method leads to a computational cost of the same order as the Multifluid with 24 sections. Actually, the two important building blocks of the CSVM are the reconstruction strategy and the convection scheme. For 1000 iterations, $185 \mathrm{~s}$ are spent for the reconstruction step, whereas $492 \mathrm{~s}$ are spent for the convection scheme out of a total of $811 \mathrm{~s}$. As these two steps are essentially made of integral evaluations, it seems clear that the last issue to be tackled now is the efficiency of the evaluation of such integrals, even if our method demonstrates its ability to reproduce the main physics of such polydisperse flows and is very promising as an extension of EMSM for industrial LES applications [34, 33]. Another solution to improve the efficiency of the method is to make use of the hybrid aspect of the method, by using more sections but a lower order reconstruction, for which analytical solutions exist.

\section{Conclusions and outcomes}

In this paper, the moment method CSVM has been introduced in order to capture polydispersion as well as to account for size-velocity correlations, based on the EMSM of [26]. Basically, whereas the initial EMSM considered only one velocity for all sizes, which is efficient for a range of small Stokes numbers, a broader spectrum of Stokes numbers can lead to very diverse size-conditioned dynamics and the necessity to be able to model such a phenomenon has become clear. We have also seen that size-conditioned dynamics can lead to particle size segregation and moment vectors very close to the frontier of the moment space, where the EM method usually experiences difficulties.

First, the EM has been improved, in order to be able to reproduce size distributions close to the frontier of the moment space. Actually, the initial algorithm proposed in [15] is able to build the NDF with canonical moments in $[0.01,0.99]$. To cover a larger part of the moment space, a Gauss-Legendre method with an adaptive support has been implemented, in such a way that quadrature points are used only where droplets lie. Furthermore, the Newton solver has been optimized by adapting the number of parameters of the EM and by tabulating the initial guess. Size-velocity correlations have been accounted for, by using one additional moment in size-velocity for each direction. Using a power reconstruction and an equilibrium constraint for droplets of zero size allows to reconstruct the velocity for each size. The evolution in phase space by drag force and evaporation has been performed by evaluating the disappearance flux at zero size and the shift in size using a quadrature method similar to CQMOM. We emphasize on the fact that combining EMSM with a quadrature approach for the phase space evolution allows to use arbitrary evaporation and drag laws. Furthermore, the proposed reconstruction is also adapted to a Multifluid Framework, leading to an adaptive method in terms of moments (one may change the number of moments or the number of sections to adapt the method to his requirements).

The evolution in physical space has been done by using a flux splitting kinetic scheme, which separates positive and negative components of the fluxes to obtain an upwind scheme. By integrating this scheme over size, a realizable scheme is obtained, which is able to reproduce the proper dynamics of each size. This method has proven to be efficient in 1D cases.

Finally, the CSVM method has been applied on three complex cases: the crossflow, the Taylor-Green vortices and the weakly turbulent free jet. Both cases are very properly reproduced by the CSVM with only 8 moments, whereas the Multifluid approach needs at least 10 sections and 30 moments to be efficient.

In future works, three issues need to be investigated. A higher order scheme is required to reduce the numerical diffusion of such a first order scheme. A second order scheme is proposed in Appendix A but higher order will be investigated. To account for the dynamics of high-Stokes-number particles in turbulent flows, higher order size-velocity moments are needed, in combination with an additional reconstruction strategy. Finally, we need to improve and optimize the integral evaluation algorithms for both reconstruction and transport in real space and compare such an approach in more realistic configurations with turbulent mixing, 
as the highly segregative cases of the present work impose to reconstruct NDF at the frontier of the moment space almost everywhere in the domain, increasing the computational cost due to the high number of iterations of the EM algorithm.

\section{Acknowledgments}

We would like to thank Damien Kah for several helpful discussions and for providing numerous inputs and code sources from his Ph.D. thesis where the EMSM was introduced. The motivation for the present study also stems from an original study of the crossflow configuration conducted in the Ph.D. Thesis of A. Vié as well as from discussions with his advisers S. Jay (IFPEN) and B. Cuenot (CERFACS), whom we take the occasion to thank here. The support from the RTRA DIGITEO as well as from Ecole Centrale Paris through the "Direction de la Recherche" for the Post-doctoral fellowship for A. Vié is gratefully acknowledged. In fact the present study received a funding from this RTRA through the MUSE project (PI M. Massot - "MUltiscale Spray combustion fully Eulerian solver in 3D : a new generation of numerical methods and algorithms, high performance simulations, validation and visualization"). The support of the France-Stanford Center for Interdisciplinary Studies through a collaborative project grant entitled "Multiscale mathematical modeling and numerical methods for multiphase and reactive flows" (PIs: P. Moin and M. Massot) has been very helpful. We also acknowledge the computational resources of the Mesocentre of Ecole Centrale Paris where some of the simulations were performed.

\section{Appendix A: A second-order robust and realizable numerical scheme}

All the results presented in the main text were obtained using a first order accurate numerical scheme for transport, because all relevant features of the CSVM may be exhibited using a low order scheme. Here we design a second order scheme, based on the work of Kah et al. [26] and the MUSCL method proposed initially by Van leer [51]. The main ingredient of a MUSCL method is the spatial reconstruction for the variables. As long as a spatial reconstruction is provided, the evolution of the conservative variables is done by assuming a piecewise constant reconstruction in half cells, each state being evaluated as the interface value of the reconstruction, and by a $2^{\text {nd }}$ order Runge-Kutta time discretization. To simplify the derivation of the scheme, integer exponents are used for the velocity reconstruction.

\section{A.1. Reconstruction strategy}

\section{A.1.1. Size moments}

To reconstruct the size moments in space, Kah et al. [26] introduced a strategy based on a limited linear reconstruction of the canonical moments (see Kah et al. [26] for more details):

$$
\begin{aligned}
M_{0, j}^{0}(x) & =M_{0, j}^{0}+D_{M_{0, j}^{0}} x \\
p_{k, j}(x) & =\bar{p}_{k, j}+D_{p_{k, j}} x
\end{aligned}
$$

So that the moments can be written as polynomials:

$$
\begin{aligned}
& M_{0}^{0}(x)=M_{0, j}^{0}+D_{M_{0, j}^{0}} x \\
& M_{0}^{1}(x)=M_{0}^{0}(x) p_{1}(x) \\
& M_{0}^{2}(x)=M_{0}^{0}(x) p_{1}(x)\left[\left(1-p_{1}(x)\right) p_{2}(x)+p_{1}\right] \\
& M_{0}^{3}(x)=M_{0}^{0}(x) p_{1}(x)\left\{\left(1-p_{1}(x)\right)\left(1-p_{2}(x)\right) p_{2}(x) p_{3}(x)+\left[\left(1-p_{1}(x)\right) p_{2}(x)+p_{1}(x)\right]^{2}\right\}
\end{aligned}
$$

We simplify the notation:

$$
M_{0}^{l}(x)=\sum_{k=0}^{N^{l}} \alpha_{k}^{l} x^{k}
$$

where $\mathbf{N}=(1,2,4,6)^{T}$ is the order of the polynomial reconstruction for each moment. 


\section{A.1.2. Size-velocity moments}

For size-velocity moments, we consider a second order polynomial reconstruction for the velocity against size for the sake of simplicity:

$$
M_{1}^{l}(x)=u_{g} \sum_{k=0}^{N^{l}} \alpha_{k}^{l} x^{k}+A_{1}(x) \sum_{k=0}^{N^{l+1}} \alpha_{k}^{l+1} x^{k}+A_{2}(x) \sum_{k=0}^{N^{l+2}} \alpha_{k}^{l+2} x^{k}
$$

where:

$$
A_{1}(x)=\bar{A}_{1, j}+D_{A_{1, j}} x, \quad A_{2}(x)=\bar{A}_{2, j}+D_{A_{2, j}} x
$$

At this point, four parameters are needed: the corrected cell values and the slope. The corrected cell values are imposed by the conservation of the cell value for each moment:

$$
\begin{aligned}
& M_{1, j}^{l}=\frac{1}{\Delta x} \int_{-\frac{\Delta x}{2}}^{\frac{\Delta x}{2}} M_{1}^{l}(x) \mathrm{d} x \\
& M_{1, j}^{l}=u_{g} M_{0, j}^{l}+\bar{A}_{1, j} M_{0, j}^{l+1}+\bar{A}_{2, j} M_{0, j}^{l+2}+D_{A_{1, j}} \gamma_{l+1}+D_{A_{2, j}} \gamma_{l+2}
\end{aligned}
$$

where:

$$
\gamma_{l}=\left[\sum_{k=0}^{N^{l}} \frac{\alpha_{k}^{l} \Delta x^{k+1}}{2^{k+2}(k+2)}\left(1-(-1)^{k+2}\right)\right]
$$

First, it can be noticed that in the case a null slope for each parameter the corrected cell value are exactly the cell value. If not, a $2 \times 2$ linear system is solved:

$$
\begin{aligned}
& \bar{A}_{1, j}=\frac{M_{0, j}^{3}\left(M_{1, j}^{0}-u_{g} M_{0, j}^{0}-D_{A_{1, j}} \gamma_{1}-D_{A_{2, j}} \gamma_{2}\right)-M_{0, j}^{2}\left(M_{1, j}^{1}-u_{g} M_{0, j}^{1}-D_{A_{1, j}} \gamma_{2}-D_{A_{2, j}} \gamma_{3}\right)}{M_{0, j}^{1} M_{0, j}^{3}-\left(M_{0, j}^{2}\right)^{2}} \\
& \bar{A}_{2, j}=\frac{M_{0, j}^{1}\left(M_{1, j}^{1}-u_{g} M_{0, j}^{1}-D_{A_{1, j}} \gamma_{2}-D_{A_{2, j}} \gamma_{3}\right)-M_{0, j}^{2}\left(M_{1, j}^{0}-u_{g} M_{0, j}^{0}-D_{A_{1, j}} \gamma_{1}-D_{A_{2, j}} \gamma_{2}\right)}{M_{0, j}^{1} M_{0, j}^{3}-\left(M_{0, j}^{2}\right)^{2}}
\end{aligned}
$$

So that the corrected cell values have a linear dependence with respect to the slopes:

$$
\begin{aligned}
& \bar{A}_{1, j}=A_{1, j}+c_{2} D_{A_{1, j}}+c_{3} D_{A_{2, j}} \\
& \bar{A}_{2, j}=A_{2, j}+d_{2} D_{A_{1, j}}+d_{3} D_{A_{2, j}}
\end{aligned}
$$

It comes that, if all the slopes are given, we can provide a conservative reconstruction of the moments, based on the canonical moments for size moments, and on the reconstruction parameters for size-velocity moments. So we need a strategy to evaluate the slopes.

\section{A.1.3. Slope evaluation}

For the canonical moments, the evaluation of the slopes is given in [26], and is based on a maximum principle that must satisfy the canonical moments. For the velocity, a maximum principle must also be satisfied at the kinetic level:

$$
r_{j}(S) \leq U(S, x) \leq R_{j}(S)
$$

where $r_{j}=\min \left(U_{j-1}(S), U_{j}(S), U_{j+1}(S)\right)$ and $R_{j}=\min \left(U_{j-1}(S), U_{j}(S), U_{j+1}(S)\right)$.

To fulfill the condition of Eq. (85), the coefficients of each polynomial are limited:

$$
\begin{aligned}
& \min \left(A_{1, j-1}, A_{1, j}, A_{1, j+1}\right) \leq \bar{A}_{1, j}+D_{A_{1, j}} x \leq \max \left(A_{1, j-1}, A_{1, j}, A_{1, j+1}\right) \\
& \min \left(A_{2, j-1}, A_{2, j}, A_{2, j+1}\right) \leq \bar{A}_{2, j}+D_{A_{2, j}} x \leq \max \left(A_{2, j-1}, A_{2, j}, A_{2, j+1}\right)
\end{aligned}
$$




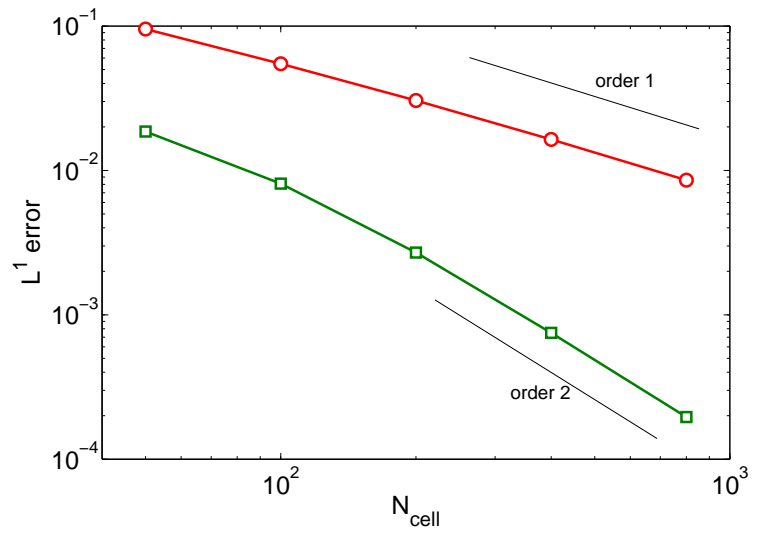

Figure 26: 1D convection: error for first (red line with circles) and second (green line with squares) order schemes

This is done by evaluating every combination of left and right slopes through linear systems, and choosing the minimum:

$$
\begin{aligned}
\left(c_{2} \pm \frac{\Delta x}{2}\right) D_{A_{1, j}}+c_{3} D_{A_{2, j}} & =\frac{-A_{1, j}+A_{1, j \pm 1}}{2} \\
d_{2} D_{A_{1, j}}+\left(d_{3} \pm \frac{\Delta x}{2}\right) D_{A_{2, j}} & =\frac{-A_{2, j}+A_{2, j \pm 1}}{2}
\end{aligned}
$$

This way, we ensure a realizable, maximum-principle-satisfying reconstruction for the whole moment set.

\section{A.2. Numerical scheme}

Using the reconstruction strategy the scheme consists in an evaluation of the interface values thanks to the reconstruction strategy. Then, at each interface, a piecewise constant convection problem is solved using a classical first order flux (Flux splitting or HLL [52] for example). The method is then embedded into a two-step Runge-Kutta temporal scheme:

$$
\begin{aligned}
\mathcal{M}_{j}^{n *} & =\mathcal{M}_{j}^{n}-\frac{1}{2} \frac{\Delta t}{\Delta x}\left(F_{j+1 / 2}\left(\mathcal{M}_{R, j}^{n} \mathcal{M}_{L, j+1}^{n}\right)-F_{j-1 / 2}\left(\mathcal{M}_{R, j-1}^{n}, \mathcal{M}_{L, j}^{n}\right)\right) \\
\mathcal{M}_{j}^{n+1} & =\mathcal{M}_{j}^{n}-\frac{\Delta t}{\Delta x}\left(F_{j+1 / 2}\left(\mathcal{M}_{R, j}^{n *}, \mathcal{M}_{L, j+1}^{n *}\right)-F_{j-1 / 2}\left(\mathcal{M}_{R, j-1}^{n *}, \mathcal{M}_{L, j}^{n *}\right)\right)
\end{aligned}
$$

where $\mathcal{M}_{j}^{n}$ is the moment vector.

\section{A.3. Application to the $1 D$ convection test case}

The second order scheme is evaluated on the test case in Sec. 6.3.1, i.e. the convection of a number density that generates a size-conditioned spatial segregation. Errors of the first order and second order schemes are compared in Fig. 26 and confirm the order of the schemes.

\section{Appendix B: Analytic solution for the 2D Crossflow}

An analytical solution is proposed for the stationary 2D crossflow configuration, based on the Lagrangian solution for each size, since no droplet crossing occurs for one size so that the Lagrangian and Eulerian approaches are equivalent. 
The Eulerian problem (2) with the monokinetic assumption $f(t, \mathbf{x}, \mathbf{v}, S)=n(t, \mathbf{x}, S) \delta(\mathbf{v}-\mathbf{u}(t, \mathbf{x}, S))$ and without evaporation is written as follows:

$$
\begin{aligned}
& \partial_{t} n+\partial_{\mathbf{x}} \cdot(n \mathbf{u})=0, \\
& \partial_{t}(n \mathbf{u})+\partial_{\mathbf{x}} \cdot(n \mathbf{u} \otimes \mathbf{u})=\frac{\mathbf{u}_{\mathbf{g}}-\mathbf{u}}{K_{d} S},
\end{aligned}
$$

with $\mathbf{u}=(U, V)$ the droplet velocity conditioned by size and $\mathbf{v}_{\mathbf{g}}=\left(U_{g}, 0\right)$ the constant gas velocity. The steady state problem is considered on the domain $\mathbf{x}=(X, Y) \in[0,+\infty[\times[0,+\infty[$ with the following conditions on boundary $Y=0$ :

$$
n(X, 0, S)=n^{0} \mathbb{1}_{\left[X_{L}, X_{R}\right]}(X), \quad U(X, 0, S)=0, \quad V(X, 0, S)=V^{0},
$$

with $n^{0}$ and $V^{0}$ some constants and with $n(X=0, Y, S)=0$ on the other incoming boundary.

Considering Lagrangian formulation, let $\left(X_{p}, Y_{p}\right)$ and $\left(U_{p}, V_{p}\right)$ be the position and the velocity of a droplet of size $S$. Its position at injection is $\left(X_{p}^{0}, 0\right)$, with $X_{L}<X_{p}^{0}<X_{R}$ and its velocity is $\left(0, V^{0}\right)$. The evolutions of the droplet characteristics is given by:

$$
\frac{d X_{p}}{d t}=U_{p}, \quad \frac{d Y_{p}}{d t}=V_{p}, \quad \frac{d U_{p}}{d t}=\frac{U_{g}-U_{p}}{K_{d} S}, \quad \frac{d V_{p}}{d t}=-\frac{V_{p}}{K_{d} S} .
$$

So, the characteristics of droplets injected at time $t=t_{0}$ are:

$$
\begin{aligned}
U_{p}(t) & =U_{g}\left[1-\exp \left(-\frac{t-t_{0}}{K_{d} S}\right)\right], & X_{p}(t) & =X_{p}^{0}+U_{g}\left\{t-t_{0}+K_{d} S\left[\exp \left(-\frac{t-t_{0}}{K_{d} S}\right)-1\right]\right\}, \\
V_{p}(t) & =V^{0} \exp \left(-\frac{t-t_{0}}{K_{d} S}\right), & Y_{p}(t) & =V^{0} K_{d} S\left[1-\exp \left(-\frac{t-t_{0}}{K_{d} S}\right)\right] .
\end{aligned}
$$

Eliminating the variable $t$, the trajectories of such droplets are then characterized by the equation:

$$
\frac{X_{p}-X_{p}^{0}}{U_{g} K_{d} S}=g\left(\frac{Y_{p}}{V^{0} K_{d} S}\right)
$$

where $g$ is the increasing function from $[0,1[$ to $[0,+\infty$ [ defined by $g(y)=-\ln (1-y)-y$. Since droplets are injected between abscissa $X_{L}$ and $X_{R}$, all droplets of size $S$ are located in $\left[X_{\min }(Y, S), X_{\max }(Y, S)\right]$, with

$$
X_{\min }(Y, S)=X_{L}+U_{g} K_{d} S g\left(\frac{Y}{V^{0} K_{d} S}\right), \quad X_{\max }(Y, S)=X_{R}+U_{g} K_{d} S g\left(\frac{Y}{V^{0} K_{d} S}\right) .
$$

The position of such droplets is plotted on Fig. 27 for two sizes. This also implies that the droplets which can reach a given position $(X, Y)$ with $X>X_{R}$ have a size $S$ such that :

$$
\frac{V^{0}}{U_{g}}\left(X-X_{R}\right) \leq h_{Y}\left(V^{0} K_{d} S\right) \leq \frac{V^{0}}{U_{g}}\left(X-X_{L}\right),
$$

where $h_{Y}$ is the decreasing function from $] Y,+\infty\left[\right.$ to $\mathbb{R}$ defined by $h_{Y}(x)=x g(Y / x)$. The size $S$ of droplets reaching $(X, Y)$ lives then in the interval $\left[S_{\min }(X, Y), S_{\max }(X, Y)\right]$, with

$$
S_{\min }(X, Y)=\frac{1}{V^{0} K_{d}} h_{Y}^{-1}\left(\frac{V^{0}}{U_{g}}\left(X-X_{L}\right)\right), \quad S_{\max }(X, Y)=\frac{1}{V^{0} K_{d}} h_{Y}^{-1}\left(\frac{V^{0}}{U_{g}}\left(X-X_{R}\right)\right) .
$$

Let us return to the Eulerian approach of system (92). The droplet velocity $(U, V)$ is given, thanks to the equivalence with the Lagrangian approach and thanks to equations (95), by

$$
U(X, Y, S)=\frac{U_{g} Y}{V^{0} K_{d} S}, \quad V(X, Y, S)=V^{0}\left(1-\frac{Y}{V^{0} K_{d} S}\right), \quad Y \in\left[0, V^{0} K_{d} S[.\right.
$$




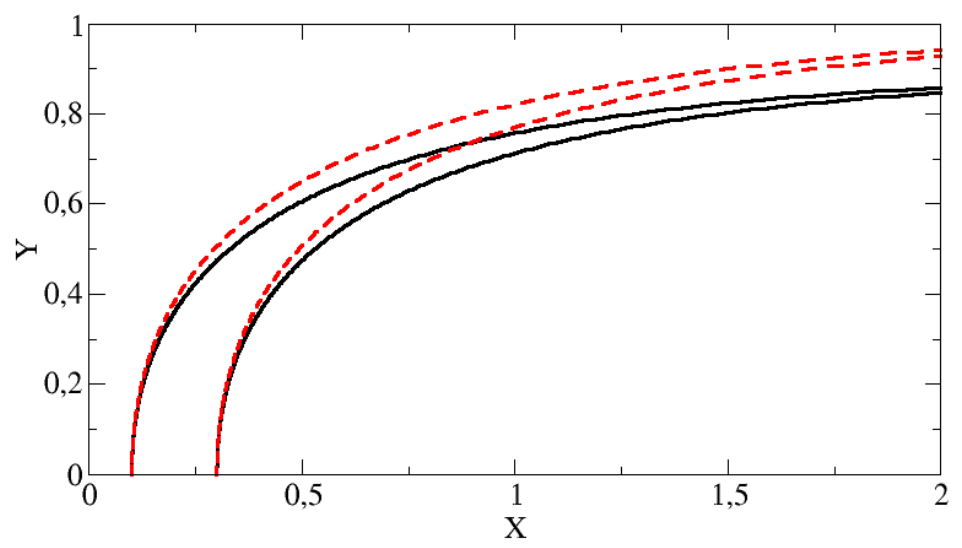

Figure 27: Position of droplets of size $S=0.9$ (between solid lines) and $S=1$ (between dashed lines).

This velocity does not depend on $X$. Moreover, the injection is uniform in the interval $\left[X_{L}, X_{R}\right]$ and the number density is independent of $X$ for $X \in\left[X_{\min }(Y, S), X_{\max }(Y, S)\right]$. The number density conservation for the size $S$ is then given by $\partial(n V) / \partial Y=0$ for $X \in\left[X_{\min }(Y, S), X_{\max }(Y, S)\right]$. Integrated between 0 and $Y$, it then induces:

$$
n(X, Y, S)=\frac{V_{0} n^{0}}{V(Y, S)}=\frac{n^{0}}{1-\frac{Y}{V^{0} K_{d} S}}, \quad Y \in\left[0, V^{0} K_{d} S\left[, \quad X \in\left[X_{\min }(Y, S), X_{\max }(Y, S)\right] .\right.\right.
$$

Equations (100) and (101) give the analytical size conditioned velocity and the NDF, solution of (92). The total number density at $(X, Y)$ plotted on Fig. 18 is then given by

$$
\begin{aligned}
& n^{t o t}(X, Y)=\int_{S_{\min (X, Y)}}^{S_{\max }(X, Y)} n(X, Y, S) \mathrm{d} S \\
& =n^{0}\left[\frac{Y}{V^{0} K_{d}} \ln \left(\frac{V^{0} K_{d} S_{\max }(X, Y)-Y}{V^{0} K_{d} S_{\min }(X, Y)-Y}\right)+S_{\max }(X, Y)-S_{\min }(X, Y)\right]
\end{aligned}
$$

and the mean surface is

$$
\begin{aligned}
S_{\text {mean }}(X, Y) & =\frac{1}{n^{\text {tot }}(X, Y)} \int_{S_{\min (X, Y)}}^{S_{\max }(X, Y)} \operatorname{Sn}(X, Y, S) \mathrm{d} S \\
& =\frac{Y}{V^{0} K_{d}}+\frac{n^{0}\left(S_{\max }(X, Y)^{2}-S_{\min }(X, Y)^{2}\right)}{2 n^{\text {tot }}(X, Y)} .
\end{aligned}
$$

\section{Appendix C: Lagrangian solution for Taylor-Green vortices}

The reference solution for the Taylor-Green vortices is chosen to be a Lagrangian computation, since there is no analytical solution to the authors' knowledge. This Lagrangian computation consists in a discrete particle simulation with a sufficiently high number of particles to achieve accurate statistics. Considering that the gas velocity $\left(U_{g}, V_{g}\right)$ is constant and given by

$$
U_{g}(x, y)=\cos (2 \pi x) \sin (2 \pi y), \quad V_{g}(x, y)=-\sin (2 \pi x) \cos (2 \pi y),
$$


the problem to solve for each numerical particle is then written:

$$
\frac{d X_{p}}{d t}=U_{p}, \quad \frac{d Y_{p}}{d t}=V_{p}, \quad \frac{d U_{p}}{d t}=\frac{U_{g}\left(X_{p}, Y_{p}\right)-U_{p}}{K_{d} S}, \quad \frac{d V_{p}}{d t}=\frac{V_{g}\left(X_{p}, Y_{p}\right)-V_{p}}{K_{d} S}
$$

for $p=1, N_{p}$ where $N_{p}$ is the number of particles. First, the initial conditions for each particle has to be given in such a way that the initial number density distribution is well reproduced. Second, a numerical scheme has to be given for the system (105).

The initial number density distribution is given by a cardinal sinus in physical space $[0,1]^{2}$ and a constant distribution in size space $[0,1]$ :

$$
\begin{aligned}
& n(x, y, S)=\frac{\sin \left(\pi W \sqrt{\left(x-x_{c}\right)^{2}+\left(y-y_{c}\right)^{2}}\right)}{\pi W \sqrt{\left(x-x_{c}\right)^{2}+\left(y-y_{c}\right)^{2}}} \quad \text { if } \quad W \sqrt{\left(x-x_{c}\right)^{2}+\left(y-y_{c}\right)^{2}} \leq 1 \\
& =0 \\
& \text { if } \quad W \sqrt{\left(x-x_{c}\right)^{2}+\left(y-y_{c}\right)^{2}}>1
\end{aligned}
$$

so that $\left[x_{c}, y_{c}\right]$ is the center of the spatial distribution of the spray and $W$ its width. To simplify, let us consider cylindrical coordinates $(r, \theta)$ around the point $\left[x_{c}, y_{c}\right]$. The initial distribution is then, as a function of these new variables: $\tilde{n}(r, \theta, S)=r \frac{\sin (\pi W r)}{\pi W r} \mathbb{1}_{[0,1 / W]}(r) \mathbb{1}_{[0,2 \pi]}(\theta) \mathbb{1}_{[0,1]}(S)$. The rejection method is used to set the numerical parcels with such a distribution. Since $\tilde{n}(r, \theta, S)$ is smaller that $\mathbb{1}_{[0,1 / W]}(r) \mathbb{1}_{[0,2 \pi]}(\theta) \mathbb{1}_{[0,1]}(S) /(\pi W)$ this method leads to the following algorithm:

1. a uniform random number generator (here the native one of fortran) is used to generate uniform random variables $r \in[0,1 / W], \theta \in[0,2 \pi], S \in[0,1]$ and $T \in[0,1]$

2a. if $\tilde{n}(r, \theta, S)>T /(\pi W)$ then the generated particle is validated,

$2 \mathrm{~b}$. otherwise the generated particle is rejected.

This algorithm is reproduced until the required number of particles is reached.

Once initial conditions are set for each particles, the Lagrangian system of equation (105) is solved in a semi-implicit manner. During one time step, the gas velocity is approximated by a constant. With this assumption, one can derive an analytical solution for position and velocity:

$$
\begin{aligned}
& U_{p}(t+\Delta t)=\left[U_{p}(t)-U_{g}\left(X_{p}(t), Y_{p}(t)\right)\right] \exp \left(-\frac{\Delta t}{K_{d} S_{p}}\right)+U_{g}\left(X_{p}(t), Y_{p}(t)\right) \\
& V_{p}(t+\Delta t)=\left[V_{p}(t)-V_{g}\left(X_{p}(t), Y_{p}(t)\right)\right] \exp \left(-\frac{\Delta t}{K_{d} S_{p}}\right)+V_{g}\left(X_{p}(t), Y_{p}(t)\right) \\
& X_{p}(t+\Delta t)=X_{p}(t)+U_{g}\left(X_{p}(t), Y_{p}(t)\right) \Delta t+K_{d} S_{p}\left[U_{p}(t)-U_{g}\left(X_{p}(t), Y_{p}(t)\right)\right]\left(1-\exp \left(-\frac{\Delta t}{K_{d} S_{p}}\right)\right) \\
& Y_{p}(t+\Delta t)=Y_{p}(t)+V_{g}\left(X_{p}(t), Y_{p}(t)\right) \Delta t+K_{d} S_{p}\left[V_{p}(t)-V_{g}\left(X_{p}(t), Y_{p}(t)\right)\right]\left(1-\exp \left(-\frac{\Delta t}{K_{d} S_{p}}\right)\right)
\end{aligned}
$$

In this approach, the only source of error is the variation of the gas velocity along the particle trajectory. This can give the intuition that this method is convergent with at least a first order accuracy. More rigorously, the convergence of the scheme is proven in what follow.

Let us denote $\left(U_{p}^{e}, V_{p}^{e}, X_{p}^{e}, Y_{p}^{e}\right)$ the exact solution of (105). For example, starting from a time $t$, the velocity in the first direction $U_{p}^{e}(t+\theta)$ is given by

$$
U_{p}^{e}(t+\theta)=U_{p}^{e}(t) \exp \left(-\frac{\theta}{K_{d} S_{p}}\right)+\int_{0}^{\theta} \frac{U_{g}\left(X_{p}^{e}(t+\tau), Y_{p}^{e}(t+\tau)\right)}{K_{d} S} \exp \left(\frac{\tau-\theta}{K_{d} S_{p}}\right) d \tau
$$

The same kind of formula can be written for the approximate solution $U_{p}(t+\theta)$, with no variation of the gas velocity:

$$
U_{p}(t+\theta)=U_{p}(t) \exp \left(-\frac{\theta}{K_{d} S_{p}}\right)+\int_{39}^{\theta} \frac{U_{g}\left(X_{p}(t), Y_{p}(t)\right)}{K_{d} S} \exp \left(\frac{\tau-\theta}{K_{d} S_{p}}\right) d \tau
$$


Moreover, the exact and approximated first coordinate is given by

$$
X_{p}^{e}(t+\tau)-X_{p}^{e}(t)=\int_{0}^{\tau} U_{p}^{e}(t+\theta) d \theta, \quad X_{p}(t+\tau)-X_{p}(t)=\int_{0}^{\tau} U_{p}(t+\theta) d \theta
$$

The same kind of formula can be written for the second direction. So the absolute error on the velocity in the first direction is such that, for $\theta>0$

$$
\begin{aligned}
\left|U_{p}^{e}(t+\theta)-U_{p}(t+\theta)\right| \leq & \left|U_{p}^{e}(t)-U_{p}(t)\right| \\
& +\frac{1}{K_{d} S} \int_{0}^{\theta}\left|U_{g}\left(X_{p}^{e}(t+\tau), Y_{p}^{e}(t+\tau)\right)-U_{g}\left(X_{p}(t), Y_{p}(t)\right)\right| \exp \left(\frac{\tau-\theta}{K_{d} S_{p}}\right) d \tau .
\end{aligned}
$$

Since the spatial gradient of the gas velocity is smaller than $2 \pi$ in both directions, and since both components of the particle velocity are smaller than one (the maximum of both components of the gas velocity), one can write

$$
\begin{aligned}
\left|U_{g}\left(X_{p}^{e}(t+\tau), Y_{p}^{e}(t+\tau)\right)-U_{g}\left(X_{p}(t), Y_{p}(t)\right)\right| & \leq 2 \pi\left(\left|X_{p}^{e}(t+\tau)-X_{p}(t)\right|+\left|Y_{p}^{e}(t+\tau)-Y_{p}(t)\right|\right) \\
& \leq 2 \pi\left(2 \tau+\left|X_{p}^{e}(t)-X_{p}(t)\right|+\left|Y_{p}^{e}(t)-Y_{p}(t)\right|\right)
\end{aligned}
$$

so that, when $\theta /\left(K_{d} S\right)$ tends to zero

$$
\left|U_{p}^{e}(t+\theta)-U_{p}(t+\theta)\right| \leq\left|U_{p}^{e}(t)-U_{p}(t)\right|+2 \pi\left(\left|X_{p}^{e}(t)-X_{p}(t)\right|+\left|Y_{p}^{e}(t)-Y_{p}(t)\right|\right)+\theta O\left(\frac{\theta}{K_{d} S}\right)
$$

and, thanks to $(110)$

$$
\begin{aligned}
\left|X_{p}^{e}(t+\Delta t)-X_{p}(t+\Delta t)\right| \leq & \left|X_{p}^{e}(t)-X_{p}(t)\right|+\int_{0}^{\Delta t}\left|U_{p}^{e}(t+\theta)-U_{p}(t+\theta)\right| d \theta \\
\leq & (1+2 \pi \Delta t)\left|X_{p}^{e}(t)-X_{p}(t)\right|+\Delta t\left|U_{p}^{e}(t)-U_{p}(t)\right|+2 \pi \Delta t\left|Y_{p}^{e}(t)-Y_{p}(t)\right| \\
& +(\Delta t)^{2} O\left(\frac{\Delta t}{K_{d} S}\right) .
\end{aligned}
$$

Thanks to a recurrence argument and thanks to equations (111) and (112) and same kind on formulas for $Y_{p}$ and $V_{p}$, one prove that the scheme is convergent with a first order accuracy. In particular, it has the great advantage of being unconditionally stable, which is also the case of the classical explicit Euler discretization.

\section{References}

[1] L. Martinez, A. Benkenida, B. Cuenot, A model for the injection boundary conditions in the context of 3D simulation of diesel spray: methodology and validation, Fuel 89(1) (2010) 219-228.

[2] D. Kah, Taking into account polydispersity in the framework of a coupled Euler-Lagrange approach for the modeling of liquid fuel injection in internal combustion engines, Ph.D. thesis, Ecole Centrale de Paris, 2010. Available online at http://tel.archives-ouvertes.fr/tel-00618786/en/.

[3] A. Vié, S. Jay, B. Cuenot, M. Massot, Accounting for polydispersion in the eulerian large eddy simulation of an aeronautical-type configuration, Submitted to Flow Turbulence and Combustion (2012) 1-38. Available online at http://hal.archives-ouvertes.fr/hal-00650605/en.

[4] F. Doisneau, F. Laurent, A. Murrone, J. Dupays, M. Massot, Eulerian multi-fluid models for the simulation of dynamics and coalescence of particles in solid propellant combustion, Submitted to J. Comput. Phys. (2012). Available online at http://hal.archives-ouvertes.fr/hal-00618806/en.

[5] F. A. Williams, Spray combustion and atomization, Phys. Fluids 1 (1958) 541-545.

[6] F. A. Williams, Combustion Theory (Combustion Science and Engineering Series), editions F.A. Williams (Reading, MA: Addison-Wesley), 1985.

[7] G. A. Bird, Molecular gas dynamics and the direct simulation of gas flows, Oxford Science Publications 42 (1994).

[8] M. Garcia, Development and validation of the Euler-Lagrange formulation on a parallel and unstructured solver for large-eddy simulation, Ph.D. thesis, Université Toulouse III, 2009. Available online at http://ethesis.inptoulouse.fr/archive/00000715/. 
[9] A. Tagliani, Hausdorff moment problem and maximum entropy: a unified approach, Appl. Math. Comput. 105 (1999) 291-305.

[10] J. Greenberg, I. Silverman, Y. Tambour, On the origin of spray sectional conservation equations, Combustion and Flame 93 (1993) 90-96.

[11] F. Laurent, M. Massot, Multi-fluid modeling of laminar poly-dispersed spray flames: origin, assumptions and comparison of the sectional and sampling methods, Combust. Theory and Modelling 5 (2001) 537-572.

[12] R. McGraw, Description of aerosol dynamics by the quadrature method of moments, Aerosol Science and Technology 27 (1997) 255-265.

[13] D. L. Marchisio, R. O. Fox, Solution of population balance equations using the direct quadrature method of moments, Journal of Aerosol Science 36 (2005) 43-73.

[14] J. Mossa, Extension polydisperse pour la description Euler-Euler des écoulements diphasiques réactifs, Ph.D. thesis, Institut National Polytechnique de Toulouse, 2005.

[15] M. Massot, F. Laurent, D. Kah, S. de Chaisemartin, A robust moment method for evaluation of the disappearance rate of evaporating sprays, SIAM J. Appl. Math. 70 (2010) 3203-3234.

[16] P. Dems, J. Carneiro, W. Polifke, Large eddy simulation of particle-laden swirling flow with a presumed function method of moments, in: $8^{t h}$ International Conference on CFD in Oil and Gas, Metallurgical and Process Industries, Trondheim, Norway, pp. 1-9.

[17] J. Carneiro, P. Dems, V. Kaufmann, W. Polifke, Eulerian simulations of polydisperse flows using a moments model with a relaxation approach for the moment transport velocities, in: International Conference on Multiphase Flow 2010, pp. $1-11$.

[18] J. Beck, A. Watkins, On the development of spray submodels based on droplet size moments, Journal of Computational Physics 182 (2002) 586-621.

[19] D. Jones, A. Watkins, Droplet size and velocity distributions for spray modelling, Journal of Computational Physics 231 (2012) 676-692.

[20] L. Fréret, O. Thomine, J. Reveillon, S. de Chaisemartin, F. Laurent, M. Massot, On the role of preferential segregation in flame dynamics in polydisperse evaporating sprays, in: Proceedings of the Summer Program 2010, Center for Turbulence Research, Stanford University, pp. 1-10.

[21] F. Laurent, Numerical analysis of eulerian multi-fluid models in the context of kinetic formulations for dilute evaporating sprays, Mathematical Modeling and Numerical Analysis 3 (2006) 431-468.

[22] G. Dufour, P. Villedieu, A second-order multi-fluid model for evaporating sprays, M2AN Math. Model. Numer. Anal. 39 (2005) 931-963.

[23] R. O. Fox, F. Laurent, M. Massot, Numerical simulation of spray coalescence in an eulerian framework: direct quadrature method of moments and multi-fluid method, Journal of Computational Physics 227 (2008) 3058-3088.

[24] L. R. Mead, N. Papanicolaou, Maximum entropy in the problem of moments, J. Math. Phys. 25 (1984) $2404-2417$.

[25] H. Dette, W. J. Studden, The theory of canonical moments with applications in statistics, probability, and analysis, Wiley Series in Probability and Statistics: Applied Probability and Statistics, John Wiley \& Sons Inc., New York, 1997. A Wiley-Interscience Publication.

[26] D. Kah, F. Laurent, M. Massot, S. Jay, A high order moment method simulating evaporation and advection of a polydisperse spray, J. Comput. Phys. (2011). Doi:10.1016/j.jcp.2011.08.032.

[27] S. Balachandar, J. Eaton, Turbulent dispersed multiphase flow, Annual Review of Fluid Mechanics 42 (2011) $111-133$.

[28] P. Février, O. Simonin, K. D. Squires, Partitioning of particle velocities in gas-solid turbulent flow into a continuous field and a spatially uncorrelated random distribution: theoretical formalism and numerical study, Journal of Fluid Mechanics $533(2005) 1-46$.

[29] C. Chalons, R. O. Fox, M. Massot, A multi-Gaussian quadrature method of moments for gas-particle flows in a LES framework, in: Proceedings of the Summer Program 2010, Center for Turbulence Research, Stanford University, Stanford, pp. 347-358.

[30] D. Kah, F. Laurent, L. Fréret, S. de Chaisemartin, R. Fox, J. Reveillon, M. Massot, Eulerian quadrature-based moment models for polydisperse evaporating sprays, Flow, Turbulence and Combustion 85 (2010) 649-676. (Special Issue Dedicated to Stephen B. Pope).

[31] A. Vié, C. Chalons, R. O. Fox, F. Laurent, M. Massot, A multi-Gaussian quadrature method of moments for simulating high-stokes-number turbulent two-phase flows, Annual Research Briefs, Center for Turbulence Research (2011) 309-320.

[32] D. L. Wright, Numerical advection of moments of the particle size distribution in Eulerian models, Journal of Aerosol Science 38 (2007) 352-369.

[33] D. Kah, M. Massot, Q. Tran, S. Jay, F. Laurent, A high order moment method with mesh movement for the description of a polydisperse evaporating spray, in: Proceedings of the International Conference on Multiphase Flows, Tampa, Florida (2010), pp. 1-15. Available online at http://hal.archives-ouvertes.fr/hal-00498214/en/.

[34] J. Bohbot, N. Gillet, A. Benkenida, IFP-C3D: an unstructured parallel solver for reactive compressible gas flow with spray, Oil \& Gas Science and Technology 64(3) (2009) 309-335.

[35] A. Vié, Simulation aux grandes échelles d' écoulements diphasiques turbulents à phase liquide dispersée, Ph.D. thesis, Institut National Polytechnique de Toulouse, 2010. Available online at http://tel.archives-ouvertes.fr/tel-00620754.

[36] V. Moureau, G. Lartigue, Y. Sommerer, C. Angelberger, O. Colin, T. Poinsot, Numerical methods for unsteady compressible multi-component reacting flows on fixed and moving grids, Journal of Computational Physics 202 (2005) $710-736$.

[37] C. Yuan, F. Laurent, R. O. Fox, An extended quadrature method of moments for population balance equations, J. Comp. Phys. 51 (2012) 1-23.

[38] J. Ferry, S. Balachandar, A fast eulerian method for disperse two-phase flow, International Journal of Multiphase Flow 
27 (2001) 1199-1226.

[39] J. Ferry, S. Balachandar, Equilibrium expansion for the eulerian velocity of small particles, Powder Technology 125 (2002) $131-139$.

[40] C. Yuan, R. Fox, Conditional quadrature method of moments for kinetic equations, Journal of Computational Physics 230(22) (2011) 8216-8246.

[41] A. Kociszewski, On the calculations of maximum entropy distributions having prescribed the moments, J. Phys.A:Math. Gen. 18 (1985) L337-L339.

[42] J. Reveillon, C. Péra, M. Massot, R. Knikker, Eulerian analysis of the dispersion of evaporating polydispersed sprays in a statistically stationary turbulent flow, Journal of Turbulence 5 (2004) 1-27.

[43] D. Wright, Numerical advection of moments of the particule size distribution in eulerian models, Journal of Aerosol Science 38(3) (2007) 352-369.

[44] M. Massot, Eulerian multi-fluid models for polydisperse evaporating sprays, volume 492 of CISM Courses and Lectures, Springer Wien New York (2007), pp. 79-123. D.L. Marchisio and R.O. Fox Editors.

[45] M. Massot, F. Laurent, S. de Chaisemartin, L. Fréret, D. Kah, Eulerian multi-fluid models: modeling and numerical methods, in: Modelling and Computation of Nanoparticles in Fluid Flows, Lectures Notes of the von Karman Institute, NATO RTO-EN-AVT-169, 2009, pp. 1-86. Available online at http://www.rta.nato.int/Pubs/RDP.asp?RDP=RTO-ENAVT-169.

[46] H. Meftah, Simulation Numérique Directe d'un spray en évaporation : Analyse et modélisation du mélange turbulent et des transferts thermiques, Ph.D. thesis, INSA de Rouen, France, 2008.

[47] J. Reveillon, DNS of Spray Combustion, Dispersion Evaporation and Combustion, volume 492 of CISM Courses and Lectures, Springer Wien New York (2007), p. 229. D.L. Marchisio and R.O. Fox Editors.

[48] J. Reveillon, F. Demoulin, Effects of the preferential segregation of droplets on evaporation and turbulent mixing, Journal of Fluid Mechanics 583 (2007) 273-302.

[49] M. Klein, A. Sadiki, J. Janicka, A digital filter based generation of inflow data for spatially developing direct numerical or large eddy simulations, J. Comput. Phys. 186 (2003) 652-665.

[50] L. Fréret, C. Lacour, S. de Chaisemartin, S. Ducruix, D. Durox, F. Laurent, M. Massot, Pulsated free jets with polydisperse spray injection : Experiments and numerical simulations, in: Proceedings of the Combustion Institute, volume 32, pp. $2215-2222$.

[51] B. van Leer, Towards the ultimate conservative difference scheme v. a second order sequel to godunov's method, J. Comput. Phys. 32 (1979) 101-136.

[52] A. Harten, P. D. Lax, B. van Leer, On upstream differencing and godunov-type schemes for hyperbolic conservation laws, SIAM Review 25 (1983) 35-61. 